\title{
The market for lawyers and quality layers in legal services
}

\author{
Elisabetta Iossa* \\ and \\ Bruno Jullien**
}

We study the functioning of the market for lawyers, considering the strategic interaction among litigants, lawyers, and judges. We investigate the value of legal representation and of systems of quality certification, such as the Queen's Counsel system. In our setting, higher quality lawyers obtain better-quality evidence and are better able to interpret it. Judges receive information from the lawyers and have reputational concerns. We show that reputational concerns generate a decision bias in favor of certified lawyers and that this causes misallocation of lawyers at the market equilibrium. As a result, whereas a higher quality of lawyers increases welfare, public information over quality may be welfare reducing.

\section{Introduction}

- Distinctive layers of quality exist within the legal profession, and it is typically observed that higher-quality lawyers are assigned higher-value cases and receive higher fees. As described, for example, by Rosen (1992) and by Garicano and Hubbard (2009a), in the United States one can distinguish between two categories of professional lawyers. The first category comprises lawyers who graduated from elite institutions, serve business clients, and charge high fees. The second category serves more individual clients and comprises lawyers who graduated from lower-tier schools, charge lower fees, and provide largely routine, noncontested legal services. Depending on their category, lawyers are then employed in different law companies, with the most reputable companies employing the most talented and well-trained lawyers. ${ }^{1}$

\footnotetext{
*DEF, University of Rome Tor Vergata, CEPR, CMPO and EIEF; elisabetta.iossa@uniroma2.it.

** Toulouse School of Economics (IDEI and GREMAQ); bruno.jullien@tse-fr.eu.

For helpful comments we wish to thank Julian Greenhill, Paul Grout, Ian Jewitt, William Kovacic, Gilat Levy, Neil Rickman, Giancarlo Spagnolo, Kathryn Spier, and seminar participants at Brunel University, Birkbeck College, Einaudi Institute for Economics and Finance, European University Institute, Paris School of Economics, University of Bristol, University of Essex, University of Rome Tor Vergata, University of Warwick, and the 2007 CMPO Workshop on the Economics of Legal Services and Justice in Bristol. Special thanks go to editor Jennifer Reinganum and two anonymous referees for their very helpful comments.

${ }^{1}$ Studying the Chicago bar, Spurr (1987) provides evidence that information, such as academic performance and quality of law school, matters, as the market tends to assign larger claims to higher-quality lawyers. The information available has improved considerably as specialized journals such as the Legal Times, American Lawyer, and National Law Journal now provide detailed information on the performance of law firms and individual lawyers.
} 
Also, in many countries, agents can choose to self-litigate rather than be represented by a professional lawyer, and the quality of self-representation is lower on average than that of professional representation because there are no entry requirements to ensure minimum quality standards. Self-litigation is cheaper and mainly used for small claims.

Distinctive layers of quality result also from the presence of systems of quality certification, such as the Queen's Counsels (QCs) system in England and Wales. QCs are lawyers who are certified to have marked themselves out in the profession; in court, they wear a distinctive uniform with a short wig and wing collar and with bands and silk gown over a special court coat. They are typically hired for more valuable cases, and from the day of their appointment they benefit from an increased fee per hour. Schemes equivalent to the QC system exist, for example, also in Scotland, Northern Ireland, Canada, New Zealand, and South Australia.

In this article, we study the functioning of the market for lawyers to analyze the value of legal representation and the desirability of quality certification systems marking distinguishable layers of quality within the legal profession. We derive the demand for lawyers and the market mechanism through which higher-quality lawyers obtain higher fees.

We build a stylized model where litigants choose whether to hire a high-quality lawyer or a low-quality lawyer (or, as alternative interpretation, they choose between professional litigation and self-litigation). We use the terms "certified" and "uncertified" to distinguish between highquality and low-quality lawyers. Certified lawyers receive a more informative signal about the state of the world and are better able to interpret the meaning of their signal than uncertified lawyers. For instance, receipt of a more informative signal may reflect better skill at generating evidence, whereas higher ability to interpret what the signal means for the case may reflect better skill at applying the relevant law to the evidence.

A dispute is resolved through an initial stage where the lawyers provide information to a lower-court judge who makes an initial decision and, if the losing litigant appeals, an appeals stage where an appeals court makes a final decision. The lower-court judge can be of two types: competent or incompetent. Competent judges are able to interpret the information provided by the lawyers, whereas incompetent judges cannot. The appeals court only comprises competent judges. Lower-court judges have private information about their type; they have reputational concerns in the sense that they wish to appear competent to an outside evaluator. In practice, judges care about how others perceive their quality, either because of a general concern for prestige or influence or because their reputation can directly influence their career and future income. Empirical evidence indeed shows that the perceived quality of judges plays an important role in their promotion to higher courts (see Miceli and Cosgel, 1994; Blanes i Vidal and Leaver, 2008; Levy, 2005; and references therein). ${ }^{2}$

In this setting, we show that the gain for a litigant from hiring a high-quality lawyer is twofold. First, it allows for a more informed appeals strategy, which leads to more efficient appeals. Second, and critical to our analysis, hiring a certified lawyer generates a decisionbias effect: incompetent judges bias their decisions in favor of certified lawyers, due to their reputational concerns. This decision-bias effect is different from the bias toward the expected shown in Ottaviani and Sørensen (2006), also present in our article. It occurs because the more informed appeals strategy of certified lawyers reveals better information about the competency of the judge. Incompetent judges then bias their decisions in favor of certified lawyers in order to minimize the risk of appeals from certified lawyers and thus the inference about their ability.

An important implication of this decision bias is that, although higher quality of lawyers increases welfare, public information over quality may be welfare reducing. The quality of lawyers improves the accuracy of the decision by two mechanisms. First, high-quality lawyers provide better-quality evidence. Second, high-quality lawyers lead to more efficient appeals. Both these

${ }^{2}$ Also, reputable judges often take prestigious and well-paid positions upon retirement from the judicials. It is not unusual, for example, for retired judges to become arbitrators in commercial disputes or international transactions. 
effects increase welfare. Instead, information over quality generates a tradeoff. On the one hand, it improves matching: in equilibrium, low-quality lawyers are hired in low-value cases and receive a basic wage, whereas high-quality lawyers are hired in high-value cases and receive a high wage. This matching effect is welfare increasing, as better legal outcomes are more valuable for high-value cases. On the other hand, public information over the quality of legal representation generates the decision bias, and this causes misallocation of lawyers at the market equilibrium: cases inefficiently arise where only one litigant hires a certified lawyer. Under some conditions, the social value of information over the quality of legal representation can then be negative. Furthermore, at the market equilibrium there is overdemand of quality; the decision-bias effect exacerbates the prisoner's dilemma problem of any legal process and results in excessive fees for lawyers. ${ }^{3}$

We also analyze the effect of quality certification on the incentives of lawyers to train and raise their ability. We show that certification helps to raise quality in the market but that free certification may lead to excessive investment by lawyers and excessive supply of certified lawyers.

Our results rationalize the perception described by respondents to a consultation paper issued by the Department of Constitutional Affairs, (2003) in the United Kingdom investigating the desirability of the QC system: "There was a perception that QCs were now instructed in circumstances where their particular skills were not really needed: for example because it might be thought that judges would pay more attention to a QC's argument, or because a simple equality of arms was needed - just because the other side had already instructed a QC." The incentives to "pay more attention" to a QC's argument can thus be seen as reflecting the incentive of less competent judges to favor litigants represented by a QC. ${ }^{4}$

In the second part of the article, we extend the model to allow for the possibility that highquality lawyers are able to "influence" the trial outcome, by raising the chance of finding evidence favorable to their case. In particular, we assume that certified lawyers generate false positives, (i.e., they may be able to obtain favorable evidence when the state of the world is unfavorable). We show that the decision-bias effect still arises and that the main qualitative insights of the basic model continue to hold at the market equilibrium. We also check the robustness of our results.

The article is organized as follows. In Section 2, we review the related literature. Section 3 presents the basic model; Sections 4 and 5 discuss the decision-making behavior of the judge and the outcome of the decision process without and with an appeals system. Section 6 studies the properties of the market for lawyers and thus the incentives to hire a certified lawyer, and the equilibrium fees. Section 7 focuses on the welfare analysis, looking at the private and social value of quality of legal services and of certification and the incentives of lawyers to train and raise their quality. Section 8 discusses the extensions on uncertain appeals and "influence" and Section 9 concludes. All proofs missing from the text are in the Appendix.

\section{Related literature}

The legal literature has long debated the impact of lawyers' capabilities in adjudication (e.g., Galanter, 1974). Consensus and evidence have been gathered on how legal representation makes for a significant difference both in the likelihood of recovery and in the amount recovered (e.g., Ross, 1970). A number of empirical articles have then analyzed the dynamics of the market for lawyers and quantified the rewards from training and specialization (Sauer, 1998; Rosen, 1992; and Pashigian, 1977; Garicano and Hubbard, 2009b). The theoretical literature on the value and

\footnotetext{
${ }^{3}$ The presence of a prisoner's dilemma in litigation was first pointed out by Ashenfelter and Bloom (1993).

${ }^{4}$ The QC system was also heavily criticized for the potential for discrimination due to a nontransparent selection process based on "secret soundings" within legal circles. Even when the appointment system was reviewed, the QC system continued to be criticized. Although it would be interesting to take the selection process into account, it would be too cumbersome to do it here and it would also add little to the present analysis, which proves the potential unsuitability of the system even when selection is not a problem.
} 
quality of legal representation, however, remains slim (see Cooter and Rubinfeld, 1989; Spier, 2007; Shavell, 2006 for a general discussion on the economics of litigation). Lawyers can affect the probability of winning through their (trial) effort (Hirshleifer and Osborne, 2001) or through their information gathering (Dewatripont and Tirole, 1999). They may then efficiently inform the court (Bundy and Elhauge, 1991) or mislead it (Kaplow and Shavell 1989). Lawyers' advice may also have no effect on adjudication in a broad set of circumstances (Che and Severinov, 2006). Our contribution to this literature is twofold. First, we derive the value of legal representation endogenously, from the interaction between litigants, lawyers, and judges, within the market mechanism, focusing on the role played by the reputational concerns of judges. ${ }^{5}$ Second, we analyze how information on the quality of legal representation affects the functioning of the market for lawyers, and thus derive policy implications on the desirability of a system of quality certification.

Our article is also related to the literature on careerist decision makers, such as regulators, managers, and experts, who try to prove their ability to make the correct decision. ${ }^{6}$ Reputational concerns may induce experts to conform to some expected behavior and disregard valuable information (e.g., Eli and Välimäki, 2003), or induce arbitrators to bias their decisions in favor of long-term players when these have biased priors (Iossa, 2007). Transparency over decisions may then be detrimental when it raises an agent's incentives to conform to some expected behavior (e.g., Prat, 2005), but it will be optimal if the expert has known bias in favor of a particular decision (Bourjade and Jullien, 2011). Career concerns may also induce experts to misreport their information (Ottaviani and Sørensen, 2006) or to bias their decisions to be more revealing (or more concealing) of their expertise (Holmström, 1999). So, for example, in Levy (2005), careerist judges contradict precedents too often in order to signal their ability, whereas in Leaver (2009), less able bureaucrats use soft policies to keep interest groups quiet and mistakes out of the public eye. Our article is the first to derive the demand for decision-making expertise and the implications of the reputational concerns on the efficiency of the equilibrium in the market for experts. We also derive the informativeness of a decision endogenously from the behavior of the informed or uninformed party (i.e., her appeal strategy). ${ }^{7}$

\section{The base model}

The general setting. We consider a setting where high-quality lawyers hold a qualification to certify that their quality is above a certain threshold. There is a mass 1 of disputes. For simplicity, we treat the occurrence of a dispute as an exogenous event and we model a dispute as a disagreement between two parties, $P 1$ and $P 2$, over the realization of a state of the world $\theta$. There are two states, $\theta=1,2$, and it is common knowledge that $\operatorname{Pr}(\theta=i)=q_{i}$. We shall refer to $q_{i}$ as the quality of the case and assume that $P 1$ has a (weakly) better case: $q_{1} \geq q_{2}$. A dispute is resolved through an initial stage where lawyers hired by parties generate and send signals (facts) to a lower-court judge who makes an initial decision and, if the losing litigant appeals, an appeals stage where the appeals court makes a final decision. Once generated, a signal needs to be interpreted to obtain relevant information.

The general structure of the game, which we explain in detail below, is the following.

(i) Disputes arise and states of the world $\theta$ are realized. The litigants sequentially choose whether to hire a certified lawyer or an uncertified lawyer ( $P 1$ chooses first).

${ }^{5}$ There is, of course, an extensive literature on the role of information and certification for consumption goods and services. References include, among others, Shapiro (1986), Biglaiser (1993), and Lizzeri (1999). These papers focus on incentives and asymmetric information issues. We abstract from these issues and focus on the interaction between the parties on a case and the behavior of the judge.

${ }^{6}$ See Bar-Isaac and Tadelis (2008) for a review of the literature on the reputational model.

${ }^{7}$ Shavell (2004) and Iossa and Palumbo (2007) discuss in depth the role of appeals as monitoring mechanisms of adjudicators, although in their models there are no reputational concerns. 
(ii) Each lawyer generates a signal and sends it to the lower-court judge. The signals transmitted are observed by the two lawyers and by the judge.

(iii) The judge makes a decision (based on his interpretation of the signals).

(iv) The losing party decides whether to appeal the judge's decision (based on his lawyer's interpretation of the signals).

(v) In the event of an appeal, the appeals court observes the state of the world and makes the final decision.

We denote by $d$ the decision of the lower-court judge and by $D$ the outcome of the appeals process. We assume that there are only two possible decisions, $d \in\{1,2\}$. A decision $d=i$ makes $P i$ win the case and it is the most appropriate in state $\theta=i$. We denote by $D=0$ the case where there are no appeals, whereas $D \in\{1,2\}$ prevails in the case of appeals.

The lawyers. There is a mass of lawyers who may work for litigants on a dispute or on some alternative activities leading to an expected utility normalized to zero. The mass is large enough for all litigants to obtain legal representation. Lawyers may be of two types, certified or uncertified, with a mass $S<2$ of certified lawyers. We endogenize $S$ in Section 7, where we consider the incentives of lawyers to become certified. We denote by $h$ (for high) the type of the certified lawyer and by $l$ (for low) the type of an uncertified lawyer. Certified lawyers are of better quality than uncertified lawyers. First, they receive a more informative signal about the state of the world. In particular, we assume that a lawyer of $P i$ generates a signal $s_{i} \in\{i, 0\}$, where $s_{i}=i$ is a perfectly informative signal that $\theta=i$. Instead, $s_{i}=0$ is bad news about $\theta=i$. A certified lawyer finds a favorable signal $s_{i}=i$ with probability 1 if $\theta=i$. An uncertified lawyer finds a favorable signal $s_{i}=i$ with probability $1-v$ if $\theta=i$, whereas with complementary probabilities he finds $s_{i}=0$. Both types of lawyer find $s_{i}=0$ with probability 1 if the state is $\theta=j$. In other words, when the state is favorable, a certified lawyer discovers it whereas an uncertified lawyer generates a false negative with probability $\nu$. When instead the state is $\theta=j$, the lawyer of $P i$ finds no favorable evidence that $\theta=i$ irrespective of his type. ${ }^{8}$

In our model, generating evidence differs from interpreting the available evidence in the light of legal principles. Faced with a signal $s_{i}$, an agent who is able to interpret it knows whether $s_{i}=i$ or $s_{i}=0$. An agent who is unable to interpret the evidence only knows $s_{i} \in\{i, 0\}$. For instance, the evidence could be a past decision and the lawyer lacks the legal knowledge to assess whether it is relevant or not for the current case. Or the evidence could be an expert report and the lawyer could be unable to assess its credibility. Certified lawyers are also better able at interpreting. To simplify, although this has no impact on our results, we assume that a certified lawyer can perfectly interpret the meaning of their signal whereas an uncertified lawyer cannot interpret it at all. ${ }^{9}$

Notice that we assume that all signals are transmitted, even when the lawyer cannot interpret it. ${ }^{10}$ This is the case if sending the signal $s_{i}$, which is favorable with some probability, is better than sending no signal, because a meaningless signal would be interpreted by the judge as bad news. We discuss this point in the benchmark section and notice here that it is consistent with the view that legal representation implies communication with the judge.

\footnotetext{
${ }^{8}$ In the working paper Iossa and Jullien (2010), we allowed for more quality levels by assuming that certified lawyers observe the state of the world with probability $r>0$. Higher $r$ captured higher quality. Results were qualitatively the same, becoming sharper with higher values of $r$. Notice that there is no loss of generality in restricting attention to only two types of lawyers, because the analysis of cases applies to any pair of lawyers. The demand for lawyers would be more complex, however.

${ }^{9}$ The analysis would be similar if the certified lawyers were only imperfectly able to interpret the signals, and/or if the uncertified lawyers had some interpretation ability. It can be shown that what matters is that the former has better ability than the latter.

${ }^{10}$ Settings where interested parties may find and misreport unfavorable information have been extensively analyzed in the literature. See, for example, Milgrom and Roberts (1986). In the present context, this issue would add little to our results whereas it would complicate the analysis significantly.
} 
In Section 8, we extend the analysis to allow for the possibility that certified lawyers alter the signal process in favor of their clients by finding false positives (i.e., by being able to present favorable evidence when the state of the world is unfavorable).

We denote by $w_{0}$ the salary of an uncertified lawyer and by $w$ the salary of a certified lawyer. $w_{0}$ is exogenously given by the productivity on some outside option. For simplicity, we set $w_{0}=0$.

The judge and the appeals court. Judges take their decisions upon receiving information (signals) by the parties and interpreting it. They have no information of their own. There are two types of lower-court judges: the competent $(C)$ and the incompetent $(I)$. Type $C$ perfectly interprets the messages sent by the lawyers, whereas type $I$ cannot interpret them. Types are private information, and we let $\gamma$ denote the proportion of competent judges. The model can be generalized to continuous types. For conciseness, we will assume throughout that

$$
\gamma<\frac{q_{2}}{1-q_{1} q_{2}}
$$

which will avoid corner equilibria (where one decision is taken with probability one by the incompetent judge) and simplify the formulas. ${ }^{11}$

The judge has reputational concerns in the sense that he wishes to appear competent to an evaluator $E$, his payoff being equal to the posterior belief $\operatorname{Pr}(C \mid$.) held by $E$ about his competency. ${ }^{12}$

We assume that the appeals court comprises only highly competent judges who observe $\theta$ and take the correct decision $D=\theta$ (the term "correct decision" will hereafter be used to denote the decision that the appeals court would take). ${ }^{13}$ This allows us to focus our attention on the reputational concerns of lower-court judges (hereafter simply referred to as the "judge") and the interplay between judges' competency and (information on) lawyer quality. We extend the analysis to allow for mistakes in appeals in Section 8.

The litigants. The litigants in a dispute (also referred to as "parties"), $P 1$ and $P 2$, obtain a zero payoff when losing the case and a payoff $V$ when winning it. The value $V$ varies across disputes and has a cumulative distribution function $F(V) .{ }^{14}$ Upon observing the judge's decision $d$, the party who loses the dispute can choose to appeal the decision. This party then incurs costs $\bar{A}$. $V$ for the appeal. ${ }^{15}$ We assume that

$$
1>\bar{A}>q_{1},
$$

which is sufficient to ensure that an uncertified lawyer never appeals and a certified lawyer appeals if and only if he knows that the decision is not correct. $\bar{A}$ is public information. We extend the analysis to the case of uncertain appeals in Section 8.

The litigants sequentially choose whether to hire a certified lawyer at salary $w$ or an uncertified lawyer at salary $w_{0}$. Sequentiality is innocuous and avoids mirror equilibria in our model. Further, in practice, the game is indeed sequential, with the plaintiff initiating the case. We assume for conciseness that $P 1$ chooses first, and then $P 2$ chooses. As explained in Section 6,

\footnotetext{
${ }^{11}$ In a previous version, Iossa and Jullien (2010), we also considered the case of corner solutions. Results were qualitatively similar to the one presented here.

${ }^{12}$ Notice that we assume that the judge does not care about his decision $d$ being correct. The analysis would extend with some altruism in the judge's preferences.

${ }^{13}$ Our results hold, provided that the appeals court comprises judges who are sufficiently more competent than lower-court ones. In practice, higher courts comprise more experienced judges or panels of judges to ensure better decision making. The higher cost of appeals courts explains why lower courts exist.

${ }^{14}$ In a previous version, Iossa and Jullien (2010), we also consider the case where there is a private bonus attached to winning in the correct state. Results are qualitatively similar to the ones presented in the present version.

${ }^{15}$ Our results would continue to hold if we assumed that appeals costs were higher for certified lawyers or that they are borne by both parties. See footnotes 18 and 22 .
} 
the reverse timing leads to the same aggregate demand and a similar welfare analysis. In order to focus on the value of legal representation, we assume away agency problems between lawyers and their clients: the hired lawyer acts in the best interest of his client and this is public information. We further assume that litigants are initially uninformed about $\theta$, thus ruling out the possibility that they attempt to signal their own information to the judge through their choice of lawyer. ${ }^{16}$

The reputation. The reputation of the judge is captured by the posterior probability that he is competent based on the observation of the decisions $d$ and $D$, whether the lawyers of the parties in the dispute are certified or not and whether they appeal. The signals sent by lawyers are excluded from the information set used to derive the reputation. ${ }^{17}$

The equilibrium concept. We use the concept of sequential equilibrium to solve the model. Beliefs are derived from players' equilibrium strategies and the strategies are rational given those beliefs. As a simplifying assumption, we shall restrict attention to equilibria such that

(i) $C$ chooses the correct decision $d^{c}=i$ when he sees a signal $s_{i}=i$; and

(ii) the decision of the judge depends only on his beliefs about $\theta$.

The first assumption rules out trivial equilibria where the judge always chooses the same decision $d=i$ and the decision $d \neq i$ would be interpreted as incompetency. The second one reflects the fact that at decision stage 2 , his beliefs about $\theta$ are the only payoff-relevant information for a judge. Equilibria satisfying the two conditions always exist.

\section{Benchmark: the judicial game without appeals}

- Consider the benchmark where there is no possibility of appeals. Here, it is never ascertained whether the decision of the judge in the lower court was correct or not; the judge's type can only be inferred from his decision.

The difference between $C$ and $I$ is that $C$ may learn the state of the world from the signals transmitted by the lawyers, whereas $I$, being unable to interpret the information, always remains uninformed. In particular, if no lawyer is certified (case $l l$ ), $C$ learns the state when one signal is favorable, $s_{i}=i$, whereas he learns nothing from the signals if $s_{1}=s_{2}=0$. If, instead, at least one lawyer is certified (cases $h l, h h$ ), the signal of this lawyer is perfectly informative about the state of the world for $C$, so $C$ is always informed.

Suppose that, when informed, $C$ takes the correct decision, that is, he takes the decision $d=i$ when he sees $s_{i}=i$. When uninformed, $C$ is in all respects like $I$, and they behave in the same way. To simplify the exposition, from now onward we refer to $C$ as the informed competent judge and to $I$ as both the incompetent judge and the uninformed competent one. We let $z_{i}$ be the probability that the judge chooses $d=i$ when he is uninformed (and thus holds beliefs $q_{i}$ ).

Using Bayes' rule, the expected payoff of $I$ when he chooses $d=i$ is given by

$$
\begin{array}{ll}
\operatorname{Pr}(C \mid d=i)=\frac{\gamma\left(q_{i}(1-v)+v z_{i}\right)}{\gamma\left(q_{i}(1-v)+v z_{i}\right)+(1-\gamma) z_{i}} & \text { for } i=1,2, \text { in case } l l \\
\operatorname{Pr}(C \mid d=i)=\frac{\gamma q_{i}}{\gamma q_{i}+(1-\gamma) z_{i}} & \text { for } i=1,2, \text { in case } h l \text { or } h h .
\end{array}
$$

Because decisions are never verified, the only (nontrivial) equilibria are such that the judge is indifferent between the two decisions. Indifference then requires that the reputation is the same for both decisions, which occurs when $I$ mimics the behavior of $C$ by randomizing in the same proportion.

\footnotetext{
${ }^{16}$ For a model that analyzes whether parties might strategically spend resources so as to signal their positive belief about the correctness of their case, see Daughety and Reinganum (2000).

${ }^{17}$ This reflects the view that the reputational concerns of the judges are with respect to the legal community, or the legal body in charge of the promotion process or even the media, which does not observe all details of the cases.
} 
Lemma 1. With no appeals, the informed competent judge chooses $d=\theta$; the incompetent judge chooses $d=i$ with probability $z_{i}=q_{i}$.

Proof. Recall that we focus on $d=\theta$ for $C$. Suppose that $z_{i}=1$. Then a decision $d \neq i$ perfectly reveals that the judge is competent and, because $\frac{\gamma q_{i}}{\gamma q_{i}+1-\gamma}<1, I$ has an incentive to deviate. Thus, the equilibrium is in mixed strategy, which requires that $\operatorname{Pr}(C \mid d=1)=\operatorname{Pr}(C \mid d=2)$. Using (3), we obtain $z_{i}=q_{i}$.

Q.E.D.

In the absence of an appeals system, the state is never revealed; whether the judge's decision is correct or not is therefore never ascertained. The reputation attached to a decision $d=1$ must therefore be the same as the reputation attached to a decision $d=2$. This in turn requires $I$ to choose $d=1$ with the same frequency as $C$, that is, with a probability of $q_{1}$. If $I$ were to choose $d=i$ with probability higher than $q_{i}$, the updating of beliefs would assign a lower reputation to a decision $d=i$ than to a decision $d \neq i$. This would generate an incentive for $I$ to deviate to $d \neq i$. Reputational concerns induce $I$ to mimic the behavior of $C$.

Remark 1. (Signal transmission) We have assumed that all signals are transmitted. We note here that it is indeed an equilibrium behavior under the following scenario. Suppose that the judge expects the transmission of information and chooses $d=i$ with probability $q_{i}$ if uninformed (as above). Let us interpret no transmission as an empty signal $s_{i}=\varnothing$. There are two possible games we can consider.

A first possibility is that $s_{i}=\varnothing$ cannot be distinguished from $s_{i}=0$. Then, $C$ would react to $s_{i}=\varnothing$ as to $s_{i}=0$ whereas $I$ would not react. Clearly, transmitting is an optimal strategy in this case, because transmitting $s_{i} \in\{0, i\}$ is weakly better than transmitting $s_{i}=0$ all the time.

The second possibility is that $s_{i}=\varnothing$ is observable by the judge. Then, no transmission would trigger some out-of-equilibrium-path reaction by the judge. Thus, it is sufficient that this reaction is unfavorable to the party not transmitting for the equilibrium with transmission to exist. A simple equilibrium behavior is $d=j$ if $i$ does not transmit. Another simple possibility is that $C$ reacts to $s_{i}=\varnothing$ as if $s_{i}=0$ and $I$ 's reaction is $\operatorname{Pr}(d=i) \leq q_{i}$.

\section{The judicial game with an appeals court}

Let us suppose now that appeals are possible and derive the behavior of the judge, given the choice of the parties as to whether to hire a certified lawyer.

Disputes with no certified lawyers. The above analysis still applies if both parties hire an uncertified lawyer. In this case, when $P i$ observes a decision $d=j$, he anticipates that the probability of reversing the decision on appeal is given by

$$
\operatorname{Pr}(\theta=j \mid d=i)=q_{j} \operatorname{Pr}\left(C, s_{1}=s_{2}=0 \mid d=i\right)+q_{j} \operatorname{Pr}(I \mid d=i),
$$

which is strictly lower than $q_{j}$ and thus than $\bar{A}$. Therefore, there is no appeal and the equilibrium is the same as in Lemma 1 . Because the state is never ascertained, reputational concerns only depend on the decision taken. This induces $I$ to mimic the behavior of $C$ and choose $d=1$ with the same frequency.

Disputes with one certified lawyer. We consider first a dispute where $P i$ hires a certified lawyer and $P j$ hires a noncertified lawyer. As pointed out above, as $C$ can always infer the state $\theta$ from the evidence shown by the certified lawyer (or the lack of evidence), he is fully informed. Consider the following strategies for the judge:

- $C$ chooses the correct decision $d^{c}=\theta$.

- $I$ randomizes between $d=1$ and $d=2$, choosing $d=i$ with probability $z_{i}$.

It can easily be proven that this strategy constitutes the optimal strategy for $C$. For conciseness, we focus here on $I$. 
Because $I$ is uninformed and he wishes to appear competent, he will arbitrage between the reputation effect in the case of no appeal and the risk that his decision is reversed on appeal, taking into account the incentives of the litigants to appeal. The appeals strategies of the two lawyers thus play an important role in determining the incentives of $I$.

The certified lawyer, interpreting the signals, knows $\theta$. He appeals $d=j$ when he has favorable evidence and only in this case. The uncertified lawyer, being uninformed, has belief facing $d=i$, given by $\operatorname{Pr}(\theta=j \mid d=i)=q_{j} \operatorname{Pr}(I \mid d=i)<\bar{A}$. Therefore, he does not appeal.

Thus, the only case of appeal occurs when the certified lawyer observes that the judge took the decision $j$ and $s_{i}=i$. Following an appeal, the expected posterior probability that the judge is competent is $\pi$. In the case where $z_{j}>0$, according to Baye's rule, an appeal reveals to $E$ that the judge is incompetent (because $d^{c}=i$ ), leading to a zero reputation $\pi=0$. Otherwise, $\pi$ is arbitrary. On the other hand, choosing $d=j$ and facing no appeal is good news. In this case, the decision is known to be correct and the reputation is $\operatorname{Pr}(C \mid d=j$, no appeal $)=\frac{\gamma}{\gamma+(1-\gamma)\left(1-z_{i}\right)}$. The expected payoff of $I$ when he chooses $d=j$ is given by

$$
\mu(d=j)=q_{i} \pi+q_{j} \frac{\gamma}{\gamma+(1-\gamma)\left(1-z_{i}\right)} .
$$

Choosing $d=i$, on the other hand, ensures that there will be no appeal and a payoff given by

$$
\mu(d=i)=\operatorname{Pr}(C \mid d=i)=\frac{\gamma q_{i}}{\gamma q_{i}+(1-\gamma) z_{i}} .
$$

Under condition (1), the equilibrium involves a mixed strategy $z_{j}>0$ and $\pi=0$, which leads to the following proposition.

Proposition 1. When party $P i$ hires a certified lawyer and $P j$ does not, in equilibrium the competent judge chooses $d=i$ with probability $q_{i}$, whereas the incompetent judge chooses $d=i$ with (unique) probability $z_{i}^{h l}$, where $q_{i}<z_{i}^{h l}<1$.

Proof. An equilibrium with $0<z_{i}^{h l}<1$ requires

$$
\frac{\gamma q_{j}}{\gamma+(1-\gamma)\left(1-z_{i}^{h l}\right)}=\frac{\gamma q_{i}}{\gamma q_{i}+(1-\gamma) z_{i}^{h l}}
$$

The LHS increases with $z_{i}$ from $\gamma q_{j}$ to $q_{j}$, whereas the RHS decreases from 1 to $\frac{\gamma q_{i}}{\gamma q_{i}+(1-\gamma)}$. A mixed-strategy equilibrium then exists if

$$
\frac{\gamma q_{i}}{\gamma q_{i}+(1-\gamma)}<q_{j}
$$

which holds for $i=1$ and $i=2$ under condition (1) because $q_{2} \leq q_{1}$. Then, $z_{i}$ solves (6a) and is given by

$$
z_{i}^{h l}=\frac{q_{i}\left(1-\gamma q_{j}\right)}{1-\gamma}>q_{i} .
$$

If condition (1) is violated, the equilibrium has $z_{i}=1$, which requires that $q_{j} \pi+q_{j}=$ $\frac{\gamma q_{i}}{\gamma q_{i}+(1-\gamma)}$. This defines a unique $\pi<1$.

Q.E.D.

Proposition 1 highlights the presence of a decision bias that arises from the interaction between the reputational concerns of judges, the appeals system, and the public nature of the quality mark. Whereas $C$ always takes the correct decision, $I$ biases his decision in favor of the party with the certified lawyer. Intuitively, the appeals strategy of a certified lawyer depends on the underlying state $\theta$, and thus reveals better information about the correctness of the judge's decision than the appeals strategy (or lack of appeals) of the uninformed lawyer. This is important, because reputational concerns exhibit some kind of risk aversion, in the sense that

$$
\operatorname{Pr}(C \mid d=i)>q_{i} \operatorname{Pr}(C \mid d=i, \theta=i)+\left(1-q_{i}\right) \operatorname{Pr}(C \mid d=i, \theta \neq i) .
$$


That is, I, ceteris paribus, prefers to play safer and choose a decision whose correctness is never ascertained rather than a decision that yields a reputational gain when found correct but a loss when found wrong. This makes $I$ prefer a decision in favor of the certified lawyer. To reestablish indifference, the reputational gain from choosing in favor of the uncertified lawyer must increase. This is only possible if $I$ chooses this decision with lower frequency than $C$, which underlies the asymmetric behavior of the judge.

The corollary below highlights the implication of an increase in the expected competency of the judge and in the quality of the case.

Corollary 1. When $P i$ hires a certified lawyer and $P j$ does not, the relative decision bias $\left(z_{i}^{h l}-\right.$ $\left.q_{i}\right) / q_{i}$ is strictly positive, increasing in the probability $\gamma$ that the judge is competent, and in the quality $q_{i}$ of the case.

Proof. From equation (7),

which gives the result.

$$
\frac{z_{i}^{h l}-q_{i}}{q_{i}}=\frac{\gamma q_{i}}{1-\gamma}
$$

The relative bias increases up to the point where $I$ always announces the decision favorable to the certified lawyer $\left(z_{i}^{h l}=1\right.$ if condition (1) is violated).

When $I$ plays safe by ruling in favor of the party with the certified lawyer, his type will not be revealed. When he rules against this party, instead, he may be revealed as incompetent but, if he turns out to be correct, his reward will be higher. The relative bias then determines the value of the safe choice as the reputation associated with $d=i$ decreases with $z_{i}^{h l} / q_{i}$. When $q_{i}$ increases, the bet on the party with the uncertified lawyer becomes less attractive, which has to be compensated by a reduction of the reputation with the safe decision.

Similarly, when $\gamma$ increases, the safe decision $d=i$ becomes relatively more attractive, as it is more likely to be generated by $C$. This must be compensated by an increase in the relative likelihood of choosing this action for $I$ so as to preserve indifference.

Disputes with two certified lawyers. Suppose now that both parties hire a certified lawyer. Both sides may appeal a wrong decision, so that the type of the judge is more likely to be revealed through the appeal. With two certified lawyers, the state is always revealed: the appeals process always reveals whether the decision is correct or not. In our setting, this creates a decision bias in favor of $P 1$ whenever $q_{1}>q_{2}$.

Proposition 2. With two certified lawyers, the competent judge chooses $d^{c}=\theta$ and the incompetent judge chooses $d=i$ with probability $z_{i}^{h h}$. When $q_{1}>q_{2}$, there is a tendency for the incompetent judge to conform in favor of the party with the better case: $q_{1}<z_{1}^{h h}<z_{1}^{h l}$ and $0<z_{2}^{h h}<q_{2}$. When $q_{1}=q_{2}$, so that cases are equally good, there is also no decision bias: $z_{i}^{h h}=q_{i}$.

Proof. The lawyer of $P i$ knows the signals and will therefore appeal an incorrect decision; no appeal will signal that the decision is correct, whereas an appeal reveals that the judge is incompetent, leading to a zero reputation. An equilibrium with $0<z_{i}^{h h}<1$ must have

$$
q_{2} \frac{\gamma}{\gamma+(1-\gamma) z_{2}^{h h}}=q_{1} \frac{\gamma}{\gamma+(1-\gamma) z_{1}^{h h}}
$$

where

$$
\operatorname{Pr}(C \mid d=i, \text { no appeal })=\frac{\gamma}{\gamma+(1-\gamma) z_{i}}
$$


The equilibrium condition then gives

$$
z_{i}^{h h}=\frac{q_{i}-\gamma q_{j}}{1-\gamma}
$$

Observe that

$$
q_{1} \leq z_{1}^{h h}=\frac{q_{1}-\gamma q_{2}}{1-\gamma}<\frac{q_{1}-\gamma q_{1} q_{2}}{1-\gamma}=z_{1}^{h l} .
$$

Because by assumption $z_{1}^{h l}<1$, it follows that $z_{2}^{h h}>0$. The equality $z_{1}^{h h}=q_{1}$ holds at $q_{1}=$ $q_{2}=1 / 2$.

Q.E.D.

With two certified lawyers, $I$ tends to favor the decision most likely to be found correct. This finding is reminiscent of the expert's model of Ottaviani and Sørensen (2006). In their setting, the state is always revealed and an expert uncertain about the state of the world shows a tendency to bias toward the expected. In our setting, with two certified lawyers the state is also fully revealed, via the appeals system. With a zero payoff attached to a decision being found incorrect on appeal, this implies that the expected reputational gain attached to a correct decision must be the same for $d=1,2$. If this were not the case, $I$ would deviate to the decision yielding a greater expected payoff. With $q_{1}>q_{2}$, we then obtain that the reputation attached to a correct decision $d=1$ must be lower than the reputation gain attached to a correct decision $d=2$. This requires $I$ to choose $d=1$ with higher frequency than $C$, that is, with probability greater than $q_{1}$. The bias disappears if $q_{1}=q_{2}$, as in this case $C$ chooses $d=1$ with the same probability as $d=2$.

Furthermore, this bias is smaller than the one arising with only one certified lawyer. In both cases, the correctness of a decision $d=2$ is always ascertained, either because the certified lawyer of $P 1$ appeals and reverses this decision or because by not appealing he reveals that the decision is correct. Instead, the correctness of a decision $d=1$ is never ascertained when the lawyer of $P 2$ is uncertified and always ascertained when he is certified. Ceteris paribus, choosing $d=1$ is therefore less risky and more rewarding for the judge when there is only one certified lawyer. Under the condition that $I$ is indifferent between $d=1$ and $d=2$, it follows that the reputational gain attached to a decision $d=2$ found correct must be higher in the setting with one certified lawyer. This requires $I$ to choose $d=2$ (resp. $d=1$ ) with lower (resp. higher) frequency when there is one certified lawyer than when there are two.

It should be apparent at this stage that the hypothesis that the uncertified lawyer never appeals is innocuous. Our qualitative results would hold provided that appeals costs are sufficiently high that the uncertified lawyers does not always appeal. We come back to this point in the extension on uncertain appeals in Section $8 .^{18}$

\section{The market for lawyers}

We now derive the demand for certified lawyers, considering the utility of a litigant in each possible scenario. Because $V$ is a scale factor, we focus on the probability of winning the case net of expected normalized appeals cost, and we denote it by $u_{i}$.

When neither party hires a certified lawyer, the probability that $P i$ wins is $q_{i}$ and the (normalized) utility of a litigant is

$$
u_{i}^{l l}=q_{i}
$$

Compared to case $l l$, hiring a certified lawyer when the other party does not affects the legal outcome when the judge is incompetent, which occurs with probability $1-\gamma$. Then the chance for the party with the certified lawyer, $P_{i}$, of obtaining a favorable decision in the lower court

${ }^{18}$ Note that if appeals costs were higher for certified lawyers, our results would continue to hold provided that the incentives of certified lawyers to appeal an incorrect decision were stronger than the incentives to appeal of the uninformed uncertified lawyers. 
changes from $q_{i}$ to $q_{i}+(1-\gamma) q_{j} z_{i}^{h l}$, as now cases will also be won whenever favored by the bias in state $j$. Furthermore, when the lower court's decision is unfavorable, the certified lawyer may discover that the decision is incorrect and appeal. The utility of the party with the certified lawyer is therefore

$$
u_{i}^{h l}=q_{i}+(1-\gamma) q_{j} z_{i}^{h l}-(1-\gamma)\left(1-z_{i}^{h l}\right) q_{i} \bar{A} .
$$

By contrast, the utility of the party $P i$ with an uncertified lawyer facing a certified lawyer is

$$
u_{i}^{l h}=q_{i}-(1-\gamma) z_{j}^{h l} q_{i} .
$$

With two certified lawyers, Proposition 2 implies that the probability that $I$ chooses $d=i$ is $z_{i}^{h h}$ and the utility of $P i$ is

$$
u_{i}^{h h}=q_{i}-(1-\gamma) z_{j}^{h h} q_{i} \bar{A} .
$$

Hiring a certified lawyer when the other side has a certified lawyer ensures that the decision is always correct. However, the appeals cost must be borne whenever I's decision is incorrect, which occurs when the state is $i$ (i.e., with probability $q_{i}$ ) but $I$ chooses $d=j$ (which occurs with probability $z_{j}^{h h}$ ).

To derive the demand for certified lawyers, we then define

$$
w_{i}^{l} \equiv u_{i}^{h l}-u_{i}^{l l} \quad \text { and } \quad w_{i}^{h} \equiv u_{i}^{h h}-u_{i}^{l h} .
$$

Thus, $w_{i}^{h}$ and $w_{i}^{l}$ capture, respectively, the gain for a party from hiring a certified lawyer when the other party has a certified lawyer and when she does not. The gain for a litigant from hiring a certified lawyer is twofold: it stems from the decision-bias effect and from more informed appeals. By hiring a certified lawyer when the opponent does not, a party gains a decision bias in her favor; when the other party also has a certified lawyer, the party gains that no decision bias against her will arise. Also, a certified lawyer discovers and appeals incorrect decisions more often than an uncertified lawyer. It is easy to show that

$$
\begin{aligned}
w_{i}^{l} & =(1-\gamma)\left(q_{j} z_{i}^{h l}-\left(1-z_{i}^{h l}\right) q_{i} \bar{A}\right) \geq 0 \\
w_{i}^{h} & \equiv(1-\gamma)\left(q_{i} z_{j}^{h l}-q_{i} z_{j}^{h h} \bar{A}\right)>0 .
\end{aligned}
$$

Taking into account the equilibrium values of $z_{i}^{h l}$, it can be shown ${ }^{19}$ that $w_{1}^{l} \geq w_{2}^{l}$ and $w_{1}^{h} \geq w_{2}^{h}$. Hiring a certified lawyer is more valuable for the party with the better case, as the bias increases with the quality of the case (Corollary 1). The party with the better case then benefits from a stronger increase in the winning probability and lower expected appeals costs as she will appeal less often.

We also have $w_{i}^{l}>w_{i}^{h} .^{20}$ This is because the costs of appeals are borne less often when the rival's lawyer is uncertified, that is, when favored by the bias than when on equal footing with the competitor. This makes it, ceteris paribus, more valuable to hire a certified lawyer when the other party does not have one.

For a given wage $w$, a party $P i$ is willing to hire a certified lawyer whenever $V w_{i}^{k} \geq w$, where $k$ refers to the behavior of the other party. Now let us define

$$
w^{l}=w_{1}^{l}>w^{h}=w_{2}^{h} .
$$

Thus, $w^{h} V$ is the highest wage at which both parties are willing to hire a certified lawyer, and $w^{l} V$ is the highest wage at which at least one party is willing to hire a certified lawyer.

\footnotetext{
${ }^{19}$ We have $w_{1}^{l}-w_{2}^{l}=\gamma\left(q_{1}-q_{2}\right)\left(\bar{A}-q_{1} q_{2} \bar{A}+q_{1} q_{2}\right)$ and $w_{1}^{h}-w_{2}^{h}=\gamma\left(q_{1}-q_{2}\right)\left(\bar{A}-q_{1} q_{2}\right)$.

${ }^{20}$ We have $w_{i}^{l}-w_{i}^{h}=\gamma q_{i} q_{j}\left(1-2 q_{j}+q_{i} \bar{A}\right)$, which is larger than $\gamma q_{i} q_{j}\left(1-q_{j}\right)^{2}$ because $\bar{A}>q_{1}$.
} 
Lemma 2. For a given wage $w$, the demand for certified lawyers is characterized as follows.

(i) if $V>\frac{w}{w^{h}}$, both sides hire a certified lawyer;

(ii) if $\frac{w}{w^{h}}>V \geq \frac{w}{w^{l}}$, only $P 1$ hires a certified lawyer;

(iii) if $\frac{w}{w^{l}}>V$, no side hires a certified lawyer.

Proof. Suppose $w<V w^{h}$. Then, $P 2$ chooses $h$ because $V w_{2}^{l}>V w_{2}^{h}>w$. The first mover chooses $h$ because it prefers to pay $w$ to induce $(h, h)$ instead of $(l, h)$. Suppose $w>V w^{l}$; then, the second mover always chooses $l$. The first mover then prefers $l$ as $V u^{l l}>V u^{h l}-w$. Suppose now that $V w^{l}>w>V w^{h}$. The second mover $P 2$ chooses $l$ if the first mover chooses $h$. The first mover $P 1$ chooses $h$ because he prefers a situation where he is the sole one with the certified lawyer to any situation where he has an uncertified lawyer: $w<V w^{l}=V\left(u_{1}^{h l}-u_{1}^{l l}\right)$ $<V\left(u_{1}^{h l}-u_{1}^{l h}\right)$.

Public information over the quality of legal service generates a " matching effect." Litigants to a dispute form different pools based on the amounts at stake: ceteris paribus, those with high-value cases hire high-quality lawyers and pay a high salary, whereas those with low-value cases hire low-quality lawyers and pay a low salary. For intermediate values of $w$, only one party hires the certified lawyer. Which party depends on the context, but under the assumption that $P 1$ moves first, he will choose to hire the lawyer.

The emergence of an asymmetric allocation of lawyers stems in our model from the decisionbias effect, which makes the value of a certified lawyer larger if the other side has none, $w_{i}^{l}>w_{i}^{h} .^{21}$ If $z_{i}^{h l}$ were equal to $q_{i}$ for $i=1,2$, as when there is no bias, then we would have $w^{l}=w^{h} .^{22}$

In the light of Lemma 2, the aggregate demand for certified lawyers is equal to

$$
D(w)=2-F\left(\frac{w}{w^{h}}\right)-F\left(\frac{w}{w^{l}}\right) .
$$

Proposition 3. There exists a unique and positive equilibrium salary $w^{*}$. It is decreasing with the cost of appeal $\bar{A}$. For $q_{1}$ not too large or $\bar{A}$ large enough, it is increasing with $\gamma$.

At the market equilibrium, demand $D(w)$ equals supply $S$. The equilibrium wage decreases with the cost of appeals, as it is borne only with a certified lawyer. For a given wage, the mass of cases with at least one certified lawyer increases when the likelihood of facing a competent judge increases. Notice that the benefits of hiring a certified lawyer is to create or correct for a bias. This is because the party does not care about when it wins but only about winning, and the better quality of the evidence provided by the lawyer affects the quality of the decision but not its probability distribution. The cost is the wage plus the expected appeals cost. Increasing judges' average competency reduces both the expected benefits from the bias and the expected appeals cost for the first certified lawyer on the case, but the latter effect dominates raising the value of the first lawyer. For the second lawyer, however, the effects are reversed and the comparison is ambiguous. But at least for $\bar{A}>q_{1}^{2} / q_{2}$, increasing $\gamma$ raises the value of the certified lawyers at all margins (marginal cases with one certified lawyer and marginal cases with two certified lawyers).

Remark 2. (Reverse timing) When $P 2$ chooses the lawyer first and $q_{2}<q_{1}$, a slight difference arises in the demand for lawyers. Considering Lemma 2, cases ( $i$ ) and ( $i i i)$ are unchanged. Also, only one party hires a certified lawyer in case $(i i)$. But the identity of the party hiring the certified lawyer depends on the sign of $V-w / w_{1}^{h}$ (remember that $w / w^{l}<w / w_{1}^{h} \leq w / w^{h}$ ). When $w / w^{l}<V<w / w_{1}^{h}$, the party hiring the certified lawyer is $P 2$ and not $P 1$. To see this, notice that in this range $P 1$ would hire a certified lawyer only if $P 2$ does not. Moreover $\left(u_{2}^{h l} V-w\right)-u_{2}^{l h} V>w_{2}^{l} V-w>0$, implying that $P 2$ prefers to be the one hiring a certified

${ }^{21}$ To see this, notice that if $z_{i}^{h l}=q_{i}$ for $i=1,2$, then $w^{l}=w^{h}$.

${ }^{22}$ Should appeals costs $\bar{B}<\bar{A}$ be borne by the party facing an appeal, results would be reinforced. One can easily check that $w^{l}$ would remain unchanged whereas $w^{h}$ would decrease, because $z_{i}^{h h}>1-z_{i}^{h l}$ makes $u^{h h}$ decrease faster with $\bar{B}$ than $u^{l h}$. Thus, $w^{l}-w^{h}$ would remain positive. 
lawyer. For $V$ above $w / w_{1}^{h}, P 1$ hires a certified lawyer in any case and, as long as $V<w / w^{h}$, $P 2$ prefers not to do it. Intuitively, this holds because hiring a certified lawyer is more valuable for the party with the better case (i.e., $P 1$ ), as explained above, and for the party moving first (because $w_{i}^{l}>w_{i}^{h}$ ). So when $P 2$ moves first, in equilibrium it may happen that she will have stronger incentives to hire the certified lawyer than $P 1$.

Notice that the aggregate demand is unchanged and the equilibrium price does not depend on the timing. The welfare analysis that follows would be similar, adjusting the formulas for the identity of the party hiring the certified lawyer (we will point to minor differences where they arise).

\section{Welfare analysis}

The value of quality. In the previous section, we have derived the gain for a litigant from hiring a certified lawyer, given the choice of the other litigant. In the corollary below we find, however, that, when it is calculated at the equilibrium, the value of quality for the parties is negative.

Corollary 2. (i) For $V \geq w / w^{h}$, each party obtains $u_{i}^{h h} V-w<u_{i}^{l l} V$. (ii) For $V \in$ $\left(w / w^{l}, w / w^{h}\right)$, party $P 1$ obtains $u_{1}^{h l} V-w>u_{1}^{l l} V$ and the other party obtains $u_{2}^{l h} V<u_{2}^{l l} V$. Moreover, $V>V\left(u_{1}^{h l}+u_{2}^{l h}\right)-w>V\left(u_{1}^{h h}+u_{2}^{h h}\right)-2 w$.

Proof. Immediate, except for the last point that follows from

$$
\begin{aligned}
u_{1}^{h l}+u_{2}^{l h} & =1-(1-\gamma)\left(1-z_{1}^{h l}\right) q_{1} \bar{A} \\
u_{1}^{h h}+u_{2}^{h h} & =1-(1-\gamma)\left(z_{2}^{h h} q_{1}+z_{1}^{h h} q_{2}\right) \bar{A},
\end{aligned}
$$

which implies $u_{1}^{h h}+u_{2}^{h h}<u_{1}^{h l}+u_{2}^{l h}$ as $z_{2}^{h h}>\left(1-z_{1}^{h l}\right)$.

There is a prisoner's dilemma problem for high $V$ : the litigants would be better off if they each hired an uncertified lawyer. Intuitively, each party has an incentive to pay a higher salary $w$ to hire a certified lawyer in order to obtain a decision bias in her favor or correct one in her disfavor. In equilibrium, both parties hire a certified lawyer and pay the additional cost $w$ but obtain no decision bias. The effects of hiring a certified lawyer are then reduced to creating an appeals cost.

Notice that the result is due to the fact that litigants attach no value to a correct decision, which would be the case if the correct decision were creating ex post efficiency gains.

Typically, society will care about the correct decision being taken. As pointed out by Shavell (1997), there is a divergence between private and social motives to use the legal system. When a party makes a litigation decision, she does not take into account the legal costs that she induces the opponent to incur (a negative externality), nor does she recognize associated effects on deterrence and other social benefits (a positive externality). Consequently, the privately determined level of litigation can either be socially excessive or inadequate. We assume, as seems reasonable, that the social benefit of a correct decision increases with the value of the dispute $V$ : a dispute between two firms over a patent worth $\$ 1$ billion will, ceteris paribus, be more relevant from a social perspective than a dispute between two neighbors over parking spaces worth $\$ 10,000$.

Let $\lambda V$ be the social value of a correct decision. ${ }^{23}$ The benefits from reversing a decision $d \neq \theta$ to $D=\theta$ through appeals is $\lambda-\bar{A}$, which we assume to be positive

$$
\lambda>\bar{A} \text {. }
$$

This implies that appeals costs are always worth incurring for society if the appeal leads to the correct decision. This seems reasonable, as otherwise it would be optimal not to have an

${ }^{23} \lambda$ can also capture the ex ante value for contractual parties of better enforcement of contractual terms (see Anderlini, Felli, and Postlelwaite, 2007, for a discussion of the role of court decisions in ex ante incentives). 
appeals system. Then, the normalized social value from the judicial procedure is $\omega V$, with $\omega=[\operatorname{Pr}(D=\theta)+\operatorname{Pr}(d=\theta, D=0)] \lambda-E(A)$, where $E(A)$ is the expected cost borne by the parties for appeals. In this function, we ignore the utility of the judge and more generally the social value of the information generated on the judge. ${ }^{24}$ The social welfare is then

$$
\Omega=\omega^{l l} \int_{0}^{\frac{w}{w^{T}}} V d F V+\omega^{h l} \int_{\frac{w}{w^{T}}}^{\frac{w}{w^{h}}} V d F(V)+\omega^{h h} \int_{\frac{w}{w^{h}}}^{\bar{V}} V d F V,
$$

where $\omega^{l l}$ denotes the value of $\omega$ for cases where no litigants hire uncertified lawyers, and so on. A first result is that quality is valuable for society, as follows.

Proposition 4. The social welfare is higher if there are certified lawyers than if all lawyers are of low quality.

Intuitively, the quality of lawyers is relevant, because it improves the accuracy of the decision by two mechanisms. First, high-quality lawyers provide better-quality evidence. Second, highquality lawyers correct wrong decisions of incompetent judges by appealing. With $\lambda>\bar{A}$, both effects improve social welfare, and hence high-quality lawyers have positive social value. ${ }^{25}$

The social value of quality certification. Although the quality of lawyers has a positive impact on welfare, this does not mean that certification leads to an efficient allocation. In this section we show that, for a given supply of high-quality lawyers, information over quality may be welfare reducing. The first question is whether certification induces a first-best allocation of lawyers.

Proposition 5. The equilibrium allocation involves too many cases with only one certified lawyer and too few cases with two certified lawyers if

$$
v<q_{1}\left(1-\frac{\bar{A}}{\lambda}\right)+\left(\frac{q_{2}-q_{1}}{2 q_{1} q_{2}}\right) \frac{\bar{A}}{\lambda} .
$$

Certification does not induce a first-best allocation of lawyers, because the decision bias makes $w^{l}>w^{h}$ and raises the mass of disputes with only one certified lawyer (Lemma 2). Consider two cases with value $V^{\prime}>V$ each with one certified lawyer. The welfare is $\omega^{h l} V+\omega^{h l} V^{\prime}$. Suppose we allocate the two certified lawyers to the case $V^{\prime}$ so as to obtain $\omega^{l l} V+\omega^{h h} V^{\prime}$. We prove in the Appendix that under condition (14), $V^{\prime}\left(\omega^{h h}-\omega^{h l}\right)>V\left(\omega^{h l}-\omega^{l l}\right)>0$, which implies that the welfare is higher when both lawyers are on the same case. ${ }^{26}$ Welfare would be maximized by allocating lawyers on the highest-value case, which would occur if there were no decision bias and $w^{l}$ were equal to $w^{h}$ (from expression (10)).

Condition (14) holds if the quality of any lawyer is not too small ( $v$ small), provided that cases are not too favorable to one party $\left(q_{1}-q_{2}\right.$ small $)$ or that the social value $\lambda$ of a correct decision is large compared to the cost of appeals. Then, in contrast with what we have seen for the private value of quality to a litigant (Lemma 2), from a social point of view the marginal value of having a certified lawyer is higher when the other party also has a certified lawyer than when she does not. This stems from the value of appeals being negative for private parties but positive for society $(-\bar{A}<0<\lambda-\bar{A})$ and from the decision bias reducing the social value of a

${ }^{24}$ Notice that in our model the judge's preferences are linear in posterior beliefs on his ability, so that his ex ante expected utility is independent of the equilibrium and equal to $\gamma$. A value of the information on the judge could be accounted for by incorporating a component $\varkappa(\pi)$ convex in $\pi$.

${ }^{25}$ If $P 2$ chooses the lawyer first, the result has to be qualified, because when only $P 2$ hires a certified lawyer, the likelihood that $I$ takes the wrong decision is larger than with no certified lawyer. But it holds if $q_{1}-q_{2}$ is small and/or $v$ and $\lambda-\bar{A}$ are not too small.

${ }^{26}$ When $P 2$ chooses first, the condition is $v<\min _{i} q_{i}\left(1-\frac{\bar{A}}{\lambda}\right)+\left(\frac{q_{i}-q_{j}}{2 q_{1} q_{2}}\right) \frac{\bar{A}}{\lambda}$. 
unique certified lawyer by lowering the likelihood that the lawyer's information will be used in the appeals process. ${ }^{27}$

To study the effect of matching and biases on the social value of certification, consider the allocation that is obtained if judges have no bias and lawyers are randomly allocated across cases. By this we mean that uninformed judges choose $d=i$ with probability $q_{i}$ and that each party has a probability $S / 2$ to be represented by a high-quality lawyer. ${ }^{28}$

Thus, a fraction $\frac{(2-S)^{2}}{4}$ of cases have no certified lawyers, a fraction $\frac{(2-S) S}{2}$ of cases have only one certified lawyer, and the remaining fraction of cases have two certified lawyers.

The welfare in the absence of a system of certification is then given by

$$
\tilde{\Omega}=\left(\frac{(2-S)^{2}}{4} \omega^{l l}+\frac{S^{2}}{4} \tilde{\omega}^{h h}+\frac{(2-S) S}{2} \widetilde{\omega}^{h l}\right) \int_{0}^{\bar{V}} V d F(V),
$$

where $\tilde{\omega}^{h h}$ and $\widetilde{\omega}^{h l}$ denote the social value for cases with, respectively, two and one certified lawyers on the case but no decision bias, that is, where $z_{i}^{h h}=z_{i}^{h l}=q_{i}$. By decomposing the welfare under certification given by $\Omega$ in expression (13), the matching effect and the decision-bias effect can be measured, respectively, by $\Omega^{m}-\tilde{\Omega}$ and $\Delta^{d b}$, where

$$
\begin{aligned}
& \Omega^{m}=\omega^{l l} \int_{0}^{\frac{w}{w^{l}}} V d F(V)+\tilde{\omega}^{h l} \int_{\frac{w}{w^{T}}}^{\frac{w}{w^{h}}} V d F(V)+\tilde{\omega}^{h h} \int_{\frac{w}{w^{h}}}^{\bar{V}} V d F(V), \\
& \Delta^{d b}=\left(\omega^{h l}-\tilde{\omega}^{h l}\right) \int_{\frac{w}{w^{T}}}^{\frac{w}{w^{h}}} V d F(V)+\left(\omega^{h h}-\tilde{\omega}^{h h}\right) \int_{\frac{w}{w^{h}}}^{V} V d F(V),
\end{aligned}
$$

and $\Omega=\Omega^{m}+\Delta^{d b}$.

Proposition 6. Suppose that $q_{1}$ is close to $1 / 2$ and $v$ is small. Then, the matching effect that arises under a system of quality certification increases social welfare, that is, $\Omega^{m}-\tilde{\Omega}>0$, whereas the decision-bias effect reduces it, that is, $\Delta^{d b}<0$.

Thus, the matching effect that arises under a system of quality certification increases social welfare if uncertified lawyers are of sufficient quality, whereas the decision-bias effect reduces it for $q_{1}$ not too large or for $v$ small enough.

Information on the quality of legal services generates a tradeoff. On the one hand, it affects the allocation of lawyers by inducing high-quality lawyers to serve clients with high-value cases. Ceteris paribus, this matching effect is welfare enhancing because high-value cases are those where a correct decision is most valuable. On the other hand, a system of certification creates a decision-bias effect that generates misallocation of lawyers, reducing the likelihood that the certified lawyer's information will be used. When this second effect prevails, a system of certification reduces social welfare. This is the case in the following example.

Corollary 3. Suppose that $v=0, q_{1}=1 / 2, V$ is uniformly distributed on $[\bar{V}-1, \bar{V}]$ where $\bar{V}$ is close to $\frac{w^{l} S}{w^{l}-w^{h}}$, and $S$ is smaller but close to 1 ; then the social welfare would be higher with no information on lawyers than with a system of certification.

The matching effect decreases in $v$, because the information available to the judge when lawyers are uncertified is more precise the smaller is $v$. The decision-bias effect is, instead, unaffected by $v$ : with one certified lawyer on the case, the information from the uncertified lawyer becomes useless to the judge. The value of certification is therefore lower when $v$ is smaller. Furthermore, under the conditions in the corollary, certification results in only cases with one certified lawyer, which exacerbates the negative impact of the decision-bias effect on the efficiency of the allocation. Moreover, this also minimizes the benefit of the matching effect:

${ }^{27}$ Notice that when a case involves only one certified lawyer, social welfare would be maximized by inducing the incompetent judge to be biased against him so as to induce efficient use of information.

${ }^{28}$ This would be the case if lawyers' quality were not observable and there were no appeals. 
as all the lawyers are assigned to $P 1$, certified lawyers are reallocated from $P 2$ to low-value cases. $^{29,30}$

Incentives to train and the supply of lawyers. Suppose there is a training stage where lawyers choose whether to train in order to raise their individual quality. There is a test certifying that quality is high. Untrained lawyers have low quality. Raising quality from low to high is costly. The cost of training is heterogeneous among lawyers, and we denote by $S(c)$ the mass of lawyers with a cost of training smaller than $c$. We assume that $S(c)$ is continuous and increasing from 0 to $\infty$ on the real line. Lawyers maximize their expected salary net of training cost. In our setup, this implies that a lawyer will either train and be certified or will not train at all, in which case his quality will be low.

In this setting, a system of certification raises quality by generating an investment effect: it induces lawyers to train to raise their salary from 0 to $w$. In particular, a lawyer chooses to train if $w-\tau-c \geq 0$, where $\tau$ is the price of a certification test. Then, the market equilibrium wage solves

$$
D(w)=S(w-\tau)
$$

and the total number of certified lawyers in the market is $S(w-\tau)$. As lawyers anticipate that quality certification brings higher income, they have incentives to make investments that enhance their legal abilities. Because investment (or equivalently certification) is costly, only the most capable lawyers obtain certification and enjoy higher equilibrium salaries.

Proposition 7 . There exists $\hat{\lambda}$ such that there is an excessive supply of certified lawyers when $\tau=0$ (free certification) if and only if $\lambda<\hat{\lambda}$. Moreover, $\hat{\lambda}>\bar{A}$ if $v \leq q_{1} \leq 2 / 3$.

Thus, for a given certification standard, whenever the social benefit of a correct decision is small, the number of lawyers who choose to invest in training is excessive compared to the social optimum. The parties have here excessive incentives to win, and hence excessive incentives to hire certified lawyers.

\section{Extensions}

- Our model has uncovered two potential biases in a judge's decision. One is due to a tendency to bias toward the expected that leads incompetent judges to favor the party most likely to win an appeal (ruling in her favor at a frequency higher than a competent judge). The second is a tendency to favor certified lawyer. This second decision bias is a source of inefficiencies not only in the judicial outcome but also in the allocation of lawyer, with too many cases with a single certified lawyer. In this extension section, we wish to study the robustness of our results on the decision bias in favor of talented lawyer. For this purpose, we neutralize the first bias by assuming that both parties are equally likely to be correct, $q_{1}=q_{2}=1 / 2$. We then study the effect of uncertainty on appeal, and extend the proof-taking dimension of quality.

${ }^{29}$ We have implicitly assumed that the information about lawyers' quality that litigants hold is the same as the one that judges hold. Although differences in practice may of course exist, it would be difficult to identify a general case. We note here, however, that the matching effect depends on the information available to litigants, whereas the decision-bias effect depends on the information available to the judge. Thus, the more (respectively less) a certification system affects the former (respectively latter), the greater the matching effect (respectively decision-bias effect) and thus the potential gain (respectively loss) from a system of certification.

${ }^{30}$ Before concluding this section, we note that in practice a lawyer's quality might be observable to the judge even when the lawyer is not certified. For instance, a lawyer on a case may have appeared before the judge on a previous occasion. The lawyer may also have appeared before other judges who may pass on their impressions, either informally as gossip or as part of the consultation process for judge appointment. However, even if judges can observe a signal of lawyer quality independently, they will still hold more precise information when quality certification is present, which suffices to lead to our qualitative results. 
Uncertain appeal. The base model has the property that, in equilibrium, an appeal reveals a judge's incompetency. This may not be the case in practice, for two reasons. First, the outcome of appeals may be uncertain; second, even a correct decision may be appealed. To address these issues, we amend the model in two directions.

Uncertainty in appeals is captured by allowing for some mistakes leading to $\operatorname{Pr}(D=\theta)=$ $1-\chi \leq 1$, where $\chi$ is small enough (in particular smaller than $1 / 2$ ). We assume that the mistakes are independent of the true underlying state of the world. This amounts to saying that appeals do not bring new evidence but simply help to reveal and interpret the evidence that was accessible to the lawyers. In particular, appealing an informed competent judge has no social value, because the judge has all the information. However, appealing when there is no certified lawyer helps uncover the information, whereas appealing an incompetent judge helps interpret the information.

Also, the appeals cost is random. It takes value $A_{i} V$, where $A_{i} \in\{0, \bar{A}\}$ and we denote by $\eta$ the probability of $A_{i}=0$. We maintain the assumption that $\bar{A}$ is large enough and $\chi$ small enough that, when the appeals cost is high, only the certified lawyer knowing that $d \neq \theta$ appeals. By contrast, an appeal always occurs when the cost is zero.

The appeals cost is private information of a party and his lawyer, so that the ex post reputation incorporates the uncertainty about the motive of the appeal.

Consider the judge's problem when both parties hire an uncertified lawyer. In this case, the previous logic applies and it is fairly easy to see that the judge's equilibrium strategy is to choose the correct decision when competent and informed, and to randomize with equal probability between the two decisions when uninformed (recall that for $q_{1}=1 / 2$ ). There is no appeal when the appeals cost is high, and in this case the judge's reputation is unchanged, at $\gamma$.

An appeal occurs when the appeals cost is zero, leading to a reputation that depends on whether the decision is upheld or reversed:

$$
\begin{aligned}
& \operatorname{Pr}(C \mid D=d=i)=\frac{\gamma((1-v)(1-\chi)+v / 2)}{\gamma((1-v)(1-\chi)+v / 2)+(1-\gamma) / 2}>\gamma \\
& \operatorname{Pr}(C \mid D \neq d=i)=\frac{\gamma((1-v) \chi+v / 2)}{\gamma((1-v) \chi+v / 2)+(1-\gamma) / 2}<\gamma .
\end{aligned}
$$

With two certified lawyers, all the information is available to the judge and the lawyers. Thus, an appeal occurs if the decision is not correct or if the appeals cost is zero. $I$ faces a probability of appeal $\eta+(1-\eta) / 2$, whereas $C$ faces only a probability $\eta$ of appeal. It is immediate to see that for $q_{1}=1 / 2$, the equilibrium entails the incompetent judge randomizing with equal proportion between the two decisions.

Let us now turn to the case where $P i$ has a certified lawyer whereas $P j$ has an uncertified lawyer. As before, we restrict attention to interior equilibria where $z_{i}<1$. The behavior of the competent judge is unchanged, because choosing the correct decision is optimal in the event where the appeals cost is zero and ensures that no appeal occurs if the appeals cost is high.

$I$ will rule in favor of the certified lawyer with probability $z_{i}$. Choosing $d=i$ ensures that an appeal only occurs with probability $\eta$, and in this case there is equal chance that the decision is upheld or reversed. We show in the Appendix that the expected payoff is then

$$
\mu(i)=\frac{(1-\eta) \gamma}{\gamma+(1-\gamma) 2 z_{i}}+\frac{\eta}{2}\left(\frac{\gamma(1-\chi)}{\gamma(1-\chi)+(1-\gamma) z_{i}}+\frac{\gamma \chi}{\gamma \chi+(1-\gamma) z_{i}}\right) .
$$

Suppose now that the judge rules against $P i$ and chooses $d=j$. In this case an appeal occurs if $A=0$ or if $\theta=i$, and thus with probability $\eta+(1-\eta) / 2$. Appeals act as a signal that the decision may not be correct and thus that the judge is incompetent. We obtain in this case a payoff

$$
\mu(j)=\frac{1}{2} \frac{(1-\eta) \gamma}{\gamma+(1-\gamma) z_{j}}+\frac{\eta}{2}\left(\frac{\gamma(1-\chi)}{\gamma(1-\chi) \frac{\eta}{\eta+(1-\eta) \chi}+(1-\gamma) z_{j}}+\frac{\gamma \chi}{\gamma \chi \frac{\eta}{1-(1-\eta) \chi}+(1-\gamma) z_{j}}\right) .
$$

An interior equilibrium is such that $\mu(i)=\mu(j)$. 
Despite the uncertainty, the main insights remain valid. Faced with diversely competent lawyers, $I$ gains from avoiding the decision being challenged by the most informed lawyer, as this would leak more information about his type. In particular, we show in the Appendix that Proposition 1 is valid; $I$ favors the certified lawyer more often than $C: 1 / 2<z_{1}^{h l}=$ $z_{2}^{h l}<1$.

By symmetry, both parties attach the same value to the certified lawyer, and we show in the Appendix that the value $w^{l}$ of a certified lawyer when the opposite party's lawyer is not certified is strictly larger than the value $w^{h}$ of a certified lawyer when the other party's lawyer is certified. Hence, the market equilibrium is the same and there is a range of intermediate cases with only one certified lawyer.

Proposition 8 . Suppose $\chi \geq 0$ and $\eta<1$. When only $P i$ hires a certified lawyer, a decision bias in favor of $P i$ arises, that is, $z^{h l}>1 / 2$. Moreover, $w^{l}>w^{h}>0$ and the equilibrium is the same as in Lemma 2. Proposition 5 holds under the condition

$$
v<\left(\frac{1-\gamma}{\gamma}\right)\left(2 z^{h l}-1\right)\left(1-\chi-\frac{\bar{A}}{\lambda}\right) \text {. }
$$

Thus, the analysis extends to this more general case.

Remark 3. The setting with uncertain appeals allows us to reemphasize that the decision bias in cases with only one certified lawyer is due to the combination of reputational concerns of judges and the transmission via the appeals system of the better information held by the certified lawyers. As in the benchmark case where there are no appeals, when appeals are free and always take place, no decision bias arises. With $\eta=1$, the better information of the certified lawyers would play no role. See the Appendix.

False positives. In what precedes, we have focused on a situation where a certified lawyer can improve the decision-making process by providing better-quality information. In legal systems, however, lawyers can also "influence" the decision by altering the signal process in favor of litigants. To capture this possibility, we now suppose that if $P i$ hires a certified lawyer, then the accuracy of the signal depends on the true state with

$$
\operatorname{Pr}\left(s_{i}=i \mid \theta=i, h\right)=1>\operatorname{Pr}\left(s_{i}=i \mid \theta=j, h\right)=v_{h}>0 .
$$

Hence, a certified lawyer can generate a false positive. We still assume that the low-quality lawyer can only produce false negatives:

$$
v_{l}=\operatorname{Pr}\left(s_{i}=0 \mid \theta=i, l\right) ; \operatorname{Pr}\left(s_{i}=i \mid \theta=j, l\right)=0 .
$$

Then, we have $\operatorname{Pr}\left(\theta=s_{i} \mid s_{i}, h\right)>1 / 2$, so that a certified lawyer alone would guide the decision, but at the same time $\operatorname{Pr}\left(s_{i}=i\right)=1 / 2+v_{h} / 2>1 / 2$, so that the certified lawyer is more likely to generate a favorable decision than the reverse. Hence, hiring a certified lawyer even without appeals would raise the chances of winning the case if there were no other lawyer.

We maintain the assumption that $\chi=\eta=0$ and $q_{1}=1 / 2$. We also preserve the equilibrium structure where $C$ chooses the decision most likely to be correct whereas $I$ randomizes, by assuming that $v_{h}$ is not too large.

Consider now the behavior of judges when $v_{h}>0$. Notice that the situation with no certified lawyers is the same as before. In the case of two certified lawyers, a repetition of our earlier analysis shows that $C$ will continue to take the correct decision conditional on the information available. A difference is that it may be the case that the information is not conclusive, that is, $s_{1}=1$ and $s_{2}=2$. In this case, $C$ randomizes with equal weight on both decisions, as does $I$. 
Whenever $P i$ hires a certified lawyer and $P j$ hires an uncertified lawyer, the correct decision is still to follow the signals when they are consistent,

$$
\begin{aligned}
\operatorname{Pr}\left(\theta=i \mid s_{i}=0\right) & =0 \\
\operatorname{Pr}\left(\theta=i \mid s_{i}=i, s_{j}=0\right) & =\frac{1}{1+v_{h} v_{l}}>\frac{1}{2},
\end{aligned}
$$

but when the two signals conflict, $C$ should follow the uncertified lawyer. Indeed,

$$
\operatorname{Pr}\left(\theta=i \mid s_{i}=i, s_{j}=j\right)=0 .
$$

As we can see, the ability to generate favorable information can backfire, in that the information of a uncertified lawyer dominates in the decision. ${ }^{31}$

Thus, $C$ chooses to rule in favor of the certified lawyer only if $s_{i}=i$ and $s_{j}=0$. This occurs with probability

$$
Q=\operatorname{Pr}\left(s_{i}=i, s_{j}=0\right)=\frac{1}{2}+\frac{v_{h} v_{l}}{2}>\frac{1}{2} .
$$

We maintain the assumption that $\bar{A}$ is large enough so that the uncertified lawyer never appeals and $\bar{A}<\frac{1}{1+v_{h} v_{l}}$, which ensures that the certified lawyer appeals $d=j$ when $s_{i}=i$ and $s_{j}=0$. The expected payoff of $I$ from choosing $d=i$ is

$$
\mu(i)=\frac{Q \gamma}{Q \gamma+(1-\gamma) z_{i}} .
$$

If, instead, he chooses $d=j$, he will be appealed by the certified lawyer of $P i$ if and only if $d=i$ is the correct decision. In this case appeal is uncertain, but the appeals decision reveals that the judge is incompetent. Hence, the reputation falls to zero following the appeals, irrespective of the outcome. ${ }^{32}$ Therefore, his payoff will be

$$
\mu(j)=\frac{(1-Q) \gamma}{\gamma+(1-\gamma) z_{j}} .
$$

As before, the difference between $\mu(i)$ and $\mu(j)$ is decreasing in $z_{i}$; further, it is now increasing in $Q$, which suggests that the equilibrium value of $z_{i}$ will be nondecreasing in $Q$. Given that the influence effect is to raise the chance of obtaining a favorable decision, in equilibrium we still have the following.

Proposition 9. Suppose $v_{h}>0$ and $v_{l}>0$. When only $P i$ hires a certified lawyer, a decision bias, in favor of $P i$ arises, that is, $z^{h l}>Q$, and is increasing with $v_{h}$ and $v_{l}$.

When certified lawyers can alter the signal process in favor of litigants, the decision-bias effect is reinforced because it is more likely that a decision against the certified lawyer triggers an appeal. Given the bias $z$, the likelihood of winning a case with a certified lawyer faced with an uncertified lawyer is

$$
Q^{h}=\gamma Q+(1-\gamma)\left(z^{h l}+\left(1-z^{h l}\right) \frac{Q}{1+v_{h} v_{l}}\right)=\gamma Q+(1-\gamma)\left(\frac{1+z^{h l}}{2}\right)>Q,
$$

where we account for the fact that the signals $\left(s_{i}=1, s_{j}=0\right)$ are not fully informative about $\theta$, hence about the outcome of the appeal.

The private value of the certified lawyer is then given by

$$
\begin{aligned}
& w^{h}=Q^{h}-\frac{1}{2}-(1-\gamma) \frac{1}{4} \bar{A} \\
& w^{l}=Q^{h}-\frac{1}{2}-(1-\gamma) Q\left(1-z^{h l}\right) \bar{A} .
\end{aligned}
$$

\footnotetext{
${ }^{31}$ A similar effect was highlighted in Shin (1998)

${ }^{32}$ This is due to our assumption that $\eta=0$.
} 
Extending the reasoning of the previous sections, we characterize the equilibrium for the case where the certified lawyer is more likely to win faced with a competent judge.

Proposition 10. Assume $v_{h} \geq 0$. Then, $w^{l}>\max \left(w^{h}, 0\right)$, and the equilibrium is the same as in Lemma 2 except that if $w^{h} \leq 0$, no case has two certified lawyers.

Thus, the analysis of the market equilibrium is similar to above. The only difference is that it could be the case that only cases with one certified lawyer occur in equilibrium.

Concerning welfare, one difference compared to the base model is that a positive signal of the certified lawyer does not reveal the state. For a competent judge and knowing the types of lawyers, the normalized value of the evidence generated is then the expectation of $\max _{k}(\operatorname{Pr}(\theta=$ $\left.\left.k \mid s_{1}, s_{2}\right)\right)$ conditional on the types of the lawyers. An appeal, on the other hand, reveals the true state, which raises the value of appeals. ${ }^{33}$

We show in the Appendix that it is still the case that $2 \omega^{h l}<\omega^{l l}+\omega^{h h}$ whenever

$$
v_{l}+v_{h}-2 v_{h} v_{l}<\left(\frac{1-\gamma}{\gamma}\right)\left(\left(2 z^{h l}-1-v_{h}\right)\left(1-\frac{\bar{A}}{\lambda}\right)+\left(1-z^{h l}\right)+(2 Q-1) \frac{\bar{A}}{\lambda}\right) .
$$

Under this condition, the optimal allocation would require that only pairs of certified lawyers be allocated to the highest-value cases. ${ }^{34}$

We should point out that the conclusions rely on $Q>1 / 2$. Although it is reasonable to assume that certified lawyers raise the chance that the proof-taking stage be favorable to the party, in a more general Bayesian setup (allowing all types of lawyers to generate false positives and false negatives), discussed in our working paper (Iossa and Jullien, 2010), it is possible that $Q<1 / 2 .{ }^{35}$ Then, new features may arise. In the case where hiring a certified lawyer would be detrimental because his ability to generate decisive evidence is not sufficient to compensate for the fact that no news is bad news, a collapse of the market for certified lawyers is possible (this occurs if $w^{l}<0$ ). It is also conceivable that $0<w^{l}<w^{h}$, in which case a symmetric allocation with only cases with zero or two certified lawyers emerges (although we could not exhibit such a situation).

\section{Conclusions}

We have studied the value of information in the quality of legal services by analyzing how quality certification affects the incentives of litigants to hire high-quality lawyers, the incentives of lawyers to invest in training, and the decision-making behavior of judges. In this context, the presence of a decision bias has adverse effect on the value of information, both for private parties and for society. We have shown that quality certification is more likely to be beneficial when the social value of a correct decision is high or when training costs are low or when the appeals cost is low.

To the extent that the social value of a correct decision is higher in systems based on precedent, such as the common law system, our results suggest that a QC system is more likely to be beneficial in a common law system than in a civil law system. This is in line with casual observation that quality certification in the form of a QC system is prevalent in countries with a common law tradition rather than in countries with a civil law tradition of codified law.

We have assumed throughout that the lower court comprises only one judge, who may be more or less competent. It would be interesting to extend the analysis to account for the

\footnotetext{
${ }^{33}$ This contrasts with the previous extension, where appeals generated a noise.

${ }^{34}$ This, however, is related to the fact that there is no appeal by uncertified lawyers. With the possibllity of small appeals costs, it is possible that $2 \omega^{h l}>\omega^{l l}+\omega^{h h}$

${ }^{35}$ In a Bayesian setting, the inability of a certified lawyer to provide favorable evidence would be interpreted in disfavor of the party, which may occur more than half the time if evidence is sparse, leading to $Q<1 / 2$. In practice, the law restricts the extent to which judges can draw negative inference from (certified) lawyers not disclosing compelling evidence favorable to their case.
} 
possibility that the lower court comprises a panel of judges and then study whether cases with certified lawyers are better adjudicated by a single judge as opposed to a panel. Two contrasting effects would play a role here. On the one hand, assigning panels to cases with one certified lawyer is beneficial because it reduces the incidence of the bias effect. On the other hand, panels are more valuable when incorrect decisions are a priori more likely, that is, when there are no certified lawyers on the case.

Finally, it would be interesting to extend the analysis to settings where litigants are heterogeneous so that, absent certification, some litigants (for instance, large corporations) are able to observe lawyers' quality better than others. In this case, a matching effect and a decisionbias effect will still be present to some extent, but a certification system may generate the additional effect of creating a level playing field among litigants. Whether or not certification will be efficient may then depend on the probability that the dispute is between asymmetric parties (say, a corporation and an individual) compared to the probability that disputes arise between symmetrical parties (say, between two corporations).

\section{Appendix}

This Appendix contains the proofs for sections 6, 7, and 8 .

Proof of Proposition 3. This follows from the fact that $D(w)$ is decreasing, $D(0)=2>S>D\left(w^{l}\right)=0$. The equilibrium salary increases with $w^{h}$ and $w^{l}$. Then,

$$
\begin{aligned}
w^{l} & =q_{1} q_{2}\left(1-\gamma q_{2}\right)-\left(q_{2}-\gamma+\gamma q_{1} q_{2}\right) q_{1} \bar{A} \\
w^{h} & \equiv q_{1} q_{2}\left(1-\gamma q_{1}\right)-q_{2}\left(q_{1}-\gamma q_{2}\right) \bar{A},
\end{aligned}
$$

which are both decreasing with $\bar{A}$. Taking the derivative, we have

$$
\frac{\partial w^{l}}{\partial \gamma}=-q_{1} q_{2}^{2}+\left(1-q_{1} q_{2}\right) q_{1} \bar{A}>-q_{1} q_{2}^{2}+\left(1-q_{1} q_{2}\right) q_{1}^{2}>0 .
$$

We also have

$$
\frac{\partial w^{h}}{\partial \gamma} \equiv-q_{1}^{2} q_{2}+q_{2}^{2} \bar{A}>0 \quad \text { if } \quad \frac{q_{1}^{2}}{q_{2}}<\bar{A} .
$$

Proof of Proposition 4. Note that

$$
\begin{aligned}
\omega^{l l} & =\left(1-(1-\gamma+\gamma \nu) 2 q_{1} q_{2}\right) \lambda ; \\
\omega^{h h} & =\lambda-(1-\gamma)\left(q_{1} z_{2}^{h h}+q_{2} z_{1}^{h h}\right) \bar{A} ; \\
\omega^{h l} & =\left(1-(1-\gamma) z_{1}^{h l} q_{2}\right) \lambda-(1-\gamma)\left(1-z_{1}^{h l}\right) q_{1} \bar{A},
\end{aligned}
$$

where, using $\lambda>\bar{A}$,

$$
\begin{aligned}
\omega^{h h}-\omega^{l l} & =\gamma \nu 2 q_{1} q_{2} \lambda+(1-\gamma)\left(2 q_{1} q_{2} \lambda-\left(q_{1} z_{2}^{h h}+q_{2} z_{1}^{h h}\right) \bar{A}\right) \\
& >(1-\gamma)\left(q_{1}-q_{2}\right)\left(z_{1}^{h h}-q_{1}\right) \lambda \geq 0
\end{aligned}
$$

and

$$
\begin{aligned}
\omega^{h l}-\omega^{l l} & =\left[\gamma \nu 2 q_{1} q_{2}+(1-\gamma)\left(2 q_{1} q_{2}-q_{2} z_{1}^{h l}-q_{1}\left(1-z_{1}^{h l}\right)\right)\right] \lambda+(1-\gamma)\left(1-z_{1}^{h l}\right) q_{1}(\lambda-\bar{A}) \\
& >(1-\gamma)\left(q_{1}-q_{2}\right)\left(z_{1}^{h l}-q_{1}\right) \lambda>0 .
\end{aligned}
$$

Thus, we find that

$$
\Omega>\omega^{l l} \int_{0}^{\bar{V}} V f(V) d V
$$

Proof of Proposition 5. To prove this result, we notice that

$$
2 \omega^{h l}-\omega^{h h}-\omega^{l l}=\gamma 2 q_{1} q_{2}\left(v \lambda-q_{1}(\lambda-\bar{A})+\left(\frac{q_{1}-q_{2}}{2 q_{1} q_{2}}\right) \bar{A}\right),
$$

which is negative under condition (14). Now consider two cases with only one certified lawyer and value $V_{1}<V_{2}$. If we reallocated the certified lawyer from the low-value case to the high-value case, we would have one case with two certified lawyers. The welfare gain would then be

$$
V_{2}\left(\omega^{h h}-\omega^{h l}\right)+V_{1}\left(\omega^{l l}-\omega^{h l}\right)>V_{2}\left(\omega^{h h}-\omega^{h l}+\omega^{l l}-\omega^{h l}\right)>0 .
$$


Hence, reducing the number of cases with one certified lawyer would be welfare improving.

Q.E.D.

Proof of Proposition 6. In the case of no bias, we have

$$
\begin{aligned}
\tilde{\omega}^{h h} & =\omega^{h h}+(1-\gamma)\left(q_{1} z_{2}^{h h}+q_{2} z_{1}^{h h}-2 q_{1} q_{2}\right) \bar{A} \\
& =\omega^{h h}-\left(q_{1}-q_{2}\right)^{2} \gamma \bar{A} \\
\tilde{\omega}^{h l} & =\left(1-(1-\gamma) q_{1} q_{2}\right) \lambda-(1-\gamma)\left(1-q_{1}\right) q_{1} \bar{A} \\
& =\omega^{h l}+\gamma q_{1}^{2}\left(q_{2} \lambda-q_{1} \bar{A}\right) .
\end{aligned}
$$

For $q_{1}$ close to $1 / 2$ and $v$ small, we have $\omega^{h h} \simeq \tilde{\omega}^{h h}$ and $\omega^{h l}-\tilde{\omega}^{h l} \simeq-\gamma(\lambda-\bar{A}) / 8$, which implies that $\Delta^{d b}<0$. Moreover, $\tilde{\omega}^{h l}$ is close to $\left(\omega^{h h}+\omega^{l l}\right) 2$, which implies

$$
\begin{aligned}
\tilde{\Omega} & \simeq \int_{0}^{\bar{V}} \omega^{l l} V d F V+\left(\frac{\omega^{h h}-\omega^{l l}}{2}\right) S \int_{0}^{\bar{V}} V d F(V) \\
\Omega^{m} & \simeq \int_{0}^{\bar{V}} \omega^{l l} V d F V+\left(\frac{\omega^{h h}-\omega^{l l}}{2}\right)\left(\int_{\frac{w}{w^{h}}}^{\bar{V}} V d F(V)+\int_{\frac{w}{w^{T}}}^{\bar{V}} V d F V\right), \\
\Omega^{m}-\tilde{\Omega} & \simeq \frac{\omega^{h h}-\omega^{l l}}{2}\left(\int_{\frac{w}{w^{h}}}^{\bar{V}} V d F(V)+\int_{\frac{w}{w^{T}}}^{\bar{V}} V d F(V)-S \int_{0}^{\bar{V}} V d F(V)\right) .
\end{aligned}
$$

Because $2-F\left(\frac{w}{w^{h}}\right)-F\left(\frac{w}{w^{l}}\right)=S$, we have

$$
S \int_{0}^{\bar{V}} V d F(V)=\left(1-F\left(\frac{w}{w^{h}}\right)\right) \int_{0}^{\bar{V}} V d F(V)+\left(1-F\left(\frac{w}{w^{l}}\right)\right) \int_{0}^{\bar{V}} V d F(V),
$$

but

$$
\frac{1}{1-F\left(\frac{w}{w^{k}}\right)} \int_{\frac{w}{w^{k}}}^{\bar{V}} V d F(V)>\int_{0}^{\bar{V}} V d F(V) ; \quad k=l, h .
$$

We thus have

$$
\int_{\frac{w}{w^{h}}}^{\bar{V}} V d F(V)+\int_{\frac{w}{w^{T}}}^{\bar{V}} V d F(V)-S \int_{0}^{\bar{V}} V d F(V)>0
$$

Given that $\omega^{h h}-\omega^{l l}>0, \Omega^{m}-\tilde{\Omega}$ is positive for $v$ small and $q_{1}$ close to $1 / 2$.

Proof of Corollary 3. First notice that when $q_{1}=1 / 2$, with no information on lawyers, there is a random allocation of lawyers and no decision bias, that is, $I$ chooses each decision with equal probability. Hence, the welfare is $\tilde{\Omega}$. For the case of a uniform distribution between $\bar{V}-1$ and $\bar{V}$, we have for an interior solution, ${ }^{36}$ using $2 \bar{V}-\frac{w}{w^{h}}-\frac{w}{w^{l}}=S$,

$$
w=\frac{w^{h} w^{l}}{w^{h}+w^{l}}(2 \bar{V}-S)
$$

The solution is interior if

$$
\begin{aligned}
\bar{V} & \geq \frac{w}{w^{h}}=\frac{w^{l}}{w^{h}+w^{l}}(2 \bar{V}-S), \\
\frac{w}{w^{l}} & =\frac{w^{h}}{w^{h}+w^{l}}(2 \bar{V}-S) \geq \bar{V}-1,
\end{aligned}
$$

which occurs for

$$
\frac{w^{l} S}{w^{l}-w^{h}}, \frac{w^{h}(1-S)+w^{l}}{w^{l}-w^{h}} \geq \bar{V}
$$

${ }^{36}$ An interior solution is such that $\bar{V} \geq \frac{w}{w^{h}}$ and $\frac{w}{w^{l}} \geq \bar{V}-1$. 
At $\frac{w^{l} S}{w^{l}-w^{h}}=\bar{V}$, and $S \leq 1$, we obtain $w=\frac{w^{l} w^{h} S}{w^{l}-w^{h}}, \bar{V}=\frac{w}{w^{h}}$, and $\bar{V}-S=\frac{w}{w^{l}}$, so that all cases have either 1 or 0 certified lawyers. Notice that this requires that $S>1-\frac{w^{h}}{w^{l}}$ for the support to be positive. Evaluating $\Omega-\tilde{\Omega}$ at $\bar{V}=\frac{w^{l} S}{w^{l}-w^{h}}$,

$$
\begin{aligned}
\Omega-\tilde{\Omega} & =\left(\omega^{h l}-\omega^{l l}\right) \int_{\bar{V}-S}^{\bar{V}} V d F(V)-\left(\frac{S^{2}}{4}\left(\omega^{h h}-\omega^{l l}\right)+\frac{(2-S) S}{2}\left(\tilde{\omega}^{h l}-\omega^{l l}\right)\right) \int_{\bar{V}-1}^{\bar{V}} V d F(V) \\
& =\left(\frac{\omega^{h h}-\omega^{l l}}{2}-\frac{\gamma}{8}(\lambda-\bar{A})\right) \int_{\bar{V}-S}^{\bar{V}} V d F(V)-\left(\frac{\omega^{h h}-\omega^{l l}}{2}\right) S \int_{\bar{V}-1}^{\bar{V}} V d F(V) \\
\Omega-\tilde{\Omega} & =\left(\left(1-\frac{3 \gamma}{2}\right) \int_{\bar{V}-S}^{\bar{V}} V d F(V)-(1-\gamma) S \int_{\bar{V}-1}^{\bar{V}} V d F(V)\right) \frac{(\lambda-\bar{A})}{4}
\end{aligned}
$$

This is negative for

$$
\left(1-\frac{3 \gamma}{2}\right) \frac{1}{S} \int_{\bar{V}-S}^{\bar{V}} V d F(V)<(1-\gamma) \int_{\bar{V}-1}^{\bar{V}} V d F(V),
$$

which holds for $S$ close to 1 because $1-3 \gamma / 2<1-\gamma$.

Proof of Proposition 7. Defining $V_{1}=\frac{w}{w^{l}}$ and $V_{2}=\frac{w}{w^{h}}$, the equilibrium conditions are

$$
\begin{gathered}
\int_{V_{1}}^{\bar{V}} f(V) d V+\int_{V_{2}}^{\bar{V}} f(V) d V=S(w-\tau) ; \\
w=w^{l} V_{1}=w^{h} V_{2},
\end{gathered}
$$

and choosing $\tau$ is equivalent to choosing the supply $S$ and consequently the wage. Indeed, we have

$$
\tau=w-W(S),
$$

where $W(S)$ defines the level of $w$ solving $S(w)=S$. Now the welfare writes as

$$
\Omega=\int_{0}^{V_{1}} \omega^{l l} V f(V) d V+\int_{V_{1}}^{V_{2}} \omega^{h l} V f(V) d V+\int_{V_{2}}^{\bar{V}} \omega^{h h} V f(V) d V-\int_{0}^{W(S)} c S^{\prime}(c) d c .
$$

Then, differentiating welfare with respect to $S$, we obtain

$$
\frac{\partial \Omega}{\partial S}=\left(\omega^{l l}-\omega^{h l}\right) V_{1} f\left(V_{1}\right) \frac{\partial V_{1}}{\partial S}+\left(\omega^{h l}-\omega^{h h}\right) V_{2} f\left(V_{2}\right) \frac{\partial V_{2}}{\partial S}-W(S),
$$

which equates the gain in judicial decisions net of the cost of additional certified lawyers. Using (A1) and (A2), the derivatives are

$$
\begin{aligned}
& \frac{\partial V_{1}}{\partial S}=\frac{-w^{h}}{f\left(V_{1}\right) w^{h}+f\left(V_{2}\right) w^{l}}, \\
& \frac{\partial V_{2}}{\partial S}=\frac{-w^{l}}{f\left(V_{1}\right) w^{h}+f\left(V_{2}\right) w^{l}},
\end{aligned}
$$

and substituting back into $\frac{\partial \Omega}{\partial S}$ we have (we use $\left.W(S)=V_{1} w^{l}=V_{2} w^{h}\right)$

$$
\left.\frac{\partial \Omega}{\partial S}\right|_{\tau=0}=\frac{f\left(V_{1}\right) w^{h}}{f\left(V_{1}\right) w^{h}+f\left(V_{2}\right) w^{l}}\left(\omega^{h l}-\omega^{l l}-w^{l}\right) V_{1}+\frac{f\left(V_{2}\right) w^{l}}{f\left(V_{1}\right) w^{h}+f\left(V_{2}\right) w^{l}}\left(\omega^{h h}-\omega^{h l}-w^{h}\right) V_{2} .
$$

Now

$$
\omega^{h l}-\omega^{l l}-w^{l}=q_{1} q_{2}\left(\left[2 \gamma \nu+1-\gamma-\gamma q_{1}\right] \lambda-\left(1-\gamma q_{2}\right)\right),
$$

which is increasing with $\lambda$ under condition (1), and

$$
\omega^{h h}-\omega^{h l}-w^{h}=q_{1} q_{2}\left(\left(1-\gamma q_{2}\right) \lambda-\left(1-\gamma q_{1}+q_{2} \gamma \bar{A}\right)\right),
$$

which is also increasing with $\lambda$. Thus, the slope is negative for $\lambda<\hat{\lambda}$, where

$$
\hat{\lambda}<\max \left(\frac{1-\gamma q_{2}}{2 \gamma \nu+1-\gamma-\gamma q_{1}}, \frac{1-\gamma q_{1}+q_{2} \gamma \bar{A}}{1-\gamma q_{2}}\right)
$$

and

$$
\hat{\lambda}>\min \left(\frac{1-\gamma q_{2}}{2 \gamma \nu+1-\gamma-\gamma q_{1}}, \frac{1-\gamma q_{1}+q_{2} \gamma \bar{A}}{1-\gamma q_{2}}\right) .
$$

Then $\frac{1-\gamma q_{2}}{2 \gamma \nu+1-\gamma-\gamma q_{1}}>1>\bar{A}$ if $q_{1}>v$.

We also have $\frac{1-\gamma q_{1}+q_{2} \gamma \bar{A}}{1-\gamma q_{2}}>\bar{A}$ if $\frac{1-\gamma q_{1}}{1-2 \gamma q_{2}}>\bar{A}$, which holds for $q_{1}<2 / 3$ as $\frac{1-\gamma q_{1}}{1-2 \gamma q_{2}}>1$. 
Proof of Proposition 8. The reputation levels are (notice they are unconditional on I)

$$
\begin{aligned}
\operatorname{Pr}(C \mid D=0, d=i) & =\frac{\gamma}{\gamma+(1-\gamma) 2 z_{i}} \\
\operatorname{Pr}(C \mid D=d=i) & =\frac{\gamma(1-\chi)}{\gamma(1-\chi)+(1-\gamma) z_{i}} \\
\operatorname{Pr}(C \mid D \neq d=i) & =\frac{\gamma \chi}{\gamma \chi+(1-\gamma) z_{i}} .
\end{aligned}
$$

Using $\operatorname{Pr}(D=0)=1-\eta, \operatorname{Pr}(D=i)=\operatorname{Pr}(D=j)=\eta / 2$, the expected payoff is then $\mu(i)$. Then,

Suppose now that the judge rules against $P i$ and chooses $d=j$. In this case, appeals occur if $A=0$ or if $\theta=i$.

$$
\begin{aligned}
& \operatorname{Pr}(D=0 \mid d=j, I)=\frac{1}{2}(1-\eta) \\
& \operatorname{Pr}(D=j \mid d=j, I)=\eta \frac{1}{2}+(1-\eta) \frac{1}{2} \chi=\frac{1}{2}(\eta+(1-\eta) \chi) \\
& \operatorname{Pr}(D=i \mid d=j, I)=\eta \frac{1}{2}+(1-\eta) \frac{1}{2}(1-\chi)=\frac{1}{2}(1-(1-\eta) \chi) .
\end{aligned}
$$

Reputation levels are

$$
\begin{aligned}
\operatorname{Pr}(C \mid D=0, d=j) & =\frac{\frac{\gamma}{2}(1-\eta)}{\frac{\gamma}{2}(1-\eta)+(1-\gamma) z_{j} \frac{(1-\eta)}{2}}=\frac{\gamma}{\gamma+(1-\gamma) z_{j}} \\
\operatorname{Pr}(C \mid D=d=j) & =\frac{\frac{\gamma}{2} \eta(1-\chi)}{\frac{\gamma}{2} \eta(1-\chi)+(1-\gamma) z_{j}\left(\eta \frac{1}{2}+(1-\eta) \frac{1}{2} \chi\right)} \\
& =\frac{\gamma \eta(1-\chi)}{\gamma \eta(1-\chi)+(1-\gamma) z_{j}(\eta+(1-\eta) \chi)} \\
\operatorname{Pr}(C \mid D \neq d=j) & =\frac{\frac{\gamma}{2} \eta \chi}{\frac{\gamma}{2} \eta \chi+(1-\gamma) z_{j}\left(\eta \frac{1}{2}+(1-\eta) \frac{1}{2}(1-\chi)\right)} \\
& =\frac{\gamma \eta \chi}{\gamma \eta \chi+(1-\gamma) z_{j}(1-(1-\eta) \chi)} .
\end{aligned}
$$

This gives a payoff $\mu(j)$ defined in (17). An interior equilibrium requires that $\mu(i)=\mu(j)$. Notice that at $z_{i}=z_{j}=1 / 2$, we have

$$
\begin{aligned}
& \mu(i)=\gamma(1-\eta)+\eta\left(\frac{\gamma(1-\chi)}{2 \gamma(1-\chi)+1-\gamma}+\frac{\gamma \chi}{2 \gamma \chi+1-\gamma}\right) \\
& \mu(j)=\frac{\gamma(1-\eta)}{1+\gamma}+\eta\left(\frac{\gamma(1-\chi)}{2 \gamma(1-\chi)\left(\frac{\eta}{\eta+(1-\eta) \chi}\right)+1-\gamma}+\frac{\gamma \chi}{2 \gamma \chi\left(\frac{\eta}{1-(1-\eta) \chi}\right)+1-\gamma}\right),
\end{aligned}
$$

which gives

$$
\begin{aligned}
\mu(i) & -\mu(j) \\
= & \gamma^{2}(1-\eta)\left(\frac{1}{1+\gamma}-2 \eta \chi(1-\chi)\right. \\
& \times\left(\frac{1-\chi}{(2 \gamma(1-\chi)+1-\gamma)(2 \gamma(1-\chi) \eta+(1-\gamma)(\eta+(1-\eta) \chi))}\right. \\
& \left.\left.+\frac{\chi}{(2 \gamma \chi+1-\gamma)(2 \gamma \chi \eta+(1-\gamma)(1-(1-\eta) \chi))}\right)\right) \\
> & \gamma^{2}(1-\eta)\left(\frac{1}{1+\gamma}-2 \chi(1-\chi)\left(\frac{1}{(2 \gamma(1-\chi)+1-\gamma)(2 \gamma(1-\chi)+(1-\gamma))}\right.\right. \\
& \left.\left.+\frac{\chi}{(2 \gamma \chi+1-\gamma)(2 \gamma \chi+(1-\gamma) \chi)}\right)\right) \\
> & \gamma^{2}(1-\eta)\left(\frac{1}{1+\gamma}-\frac{2 \chi(1-\chi)}{1+\gamma}\left(\frac{1}{2 \gamma(1-\chi)+1-\gamma}+\frac{1}{2 \gamma \chi+1-\gamma}\right)\right) \\
> & \gamma^{2}(1-\eta)\left(\frac{1}{1+\gamma}-\frac{4 \chi(1-\chi)}{1+\gamma}\right)>0,
\end{aligned}
$$

(C) RAND 2012 
where we use $\frac{1-\chi}{2 \gamma(1-\chi)+1-\gamma}<\frac{1}{1+\gamma}$ and $\max _{\chi} \frac{1}{2 \gamma(1-\chi)+1-\gamma}+\frac{1}{2 \gamma \chi+1-\gamma}=2$. Thus, the value $\mu(j)$ is smaller than $\mu(i)$ at $z_{i}=1 / 2$, due to $\eta<1$ (with $\eta=1$, we find $\mu(j)=\mu(i)$ at $z_{i}=1 / 2$ ). Moreover, $\mu(i)-\mu(j)$ decreases with $z_{i}$. Hence, we conclude that there exists a unique equilibrium value $z^{h l}$ larger than $1 / 2$. For this value to be less than 1 , it must be the case that $\mu(i)<\mu(j)$ at $z_{i}=1$, that is,

$$
(1-\eta) \frac{2 \gamma}{2-\gamma}+\eta\left(\frac{\gamma(1-\chi)}{\gamma(1-\chi)+1-\gamma}+\frac{\gamma \chi}{\gamma \chi+1-\gamma}\right)<1
$$

This rewrites as

$$
(1-\eta) \frac{2 \gamma}{2-\gamma}+\eta \gamma\left(\frac{1-\gamma+2 \gamma \chi(1-\chi)}{1-\gamma+\gamma^{2} \chi(1-\chi)}\right)<1
$$

But

$$
\gamma\left(\frac{1-\gamma+2 \gamma \chi(1-\chi)}{1-\gamma+\gamma^{2} \chi(1-\chi)}\right) \in\left[\gamma, \frac{2 \gamma}{2-\gamma}\right]
$$

and hence the condition holds under condition (1). By symmetry, we have $z_{1}^{h l}=z_{2}^{h l}$. Therefore, we drop the index $i$ from now on. Then we have

$$
\begin{aligned}
u^{l l} & =1 / 2 \\
u^{h l} & =u^{l l}+(1-\eta)(1-\gamma)\left(z^{h l}-\frac{1}{2}+\left(1-z^{h l}\right) \frac{1}{2}(1-\chi-\bar{A})\right) \\
& =u^{l l}+(1-\eta)(1-\gamma)\left(\frac{z^{h l}}{2}-\left(1-z^{h l}\right) \frac{1}{2}(\chi+\bar{A})\right) \\
u^{l h} & =u^{l l}-(1-\eta)(1-\gamma)\left(\frac{z^{h l}}{2}-\left(1-z^{h l}\right) \frac{1}{2} \chi\right) .
\end{aligned}
$$

By symmetry again, each party attaches the same value for the certified lawyer,

$$
\begin{aligned}
& w^{l}=(1-\eta)(1-\gamma)\left(\frac{z^{h l}}{2}-\left(\frac{1-z^{h l}}{2}\right) \chi-\left(\frac{1-z^{h l}}{2}\right) \bar{A}\right) \\
& w^{h} \equiv(1-\eta)(1-\gamma)\left(\frac{z^{h l}}{2}-\left(\frac{1-z^{h l}}{2}\right) \chi-\frac{\bar{A}}{4}\right) .
\end{aligned}
$$

Due to $z^{h l}>1 / 2$, we find again that $w^{l}>w^{h}$. Hence, the market equilibrium analysis and Lemma 2 are valid.

Finally, the welfares

$$
\begin{aligned}
\omega^{l l}= & \left(\eta(1-\chi)+(1-\eta)\left(\gamma\left(1-v+\frac{v}{2}\right)+(1-\gamma) \frac{1}{2}\right)\right) \lambda \\
\omega^{h h}= & \left(\eta(1-\chi)+(1-\eta)\left(\gamma+(1-\gamma) \frac{1}{2}+(1-\gamma) \frac{1-\chi}{2}\right)\right) \lambda-(1-\eta)(1-\gamma) \frac{\bar{A}}{2} \\
= & \omega^{l l}+(1-\eta) \gamma \frac{\nu}{2}+(1-\eta)(1-\gamma) \frac{1}{2}((1-\chi) \lambda-\bar{A})>\omega^{l l} \\
\omega^{h l}= & \left(\eta(1-\chi)+(1-\eta)\left(\gamma+(1-\gamma) \frac{1}{2} z^{h l}+(1-\gamma)\left(1-z^{h l}\right)\left(\frac{1}{2}+\frac{1-\chi}{2}\right)\right)\right) \lambda+ \\
& -(1-\eta)(1-\gamma)\left(\frac{1-z^{h l}}{2}\right) \bar{A} \\
= & \omega^{l l}+(1-\eta) \frac{\gamma v}{2} \lambda+(1-\eta)(1-\gamma)\left(1-z^{h l}\right) \frac{1}{2}((1-\chi) \lambda-\bar{A})>\omega^{l l} \\
\omega^{h l}= & \gamma \lambda+(1-\gamma) z_{1}^{h l} q_{1} \lambda+(1-\gamma)\left(1-z_{1}^{h l}\right) \lambda-(1-\gamma)\left(1-z_{1}^{h l}\right) q_{1} \bar{A} \\
= & \left(1-(1-\gamma) z_{1}^{h l} q_{2}\right) \lambda-(1-\gamma)\left(1-z_{1}^{h l}\right) q_{1} \bar{A} .
\end{aligned}
$$

Thus, the presence of certified lawyers is valuable (Proposition 4).

Moreover,

$$
2 \omega^{h l}-\omega^{h h}-\omega^{l l}=(1-\eta) \frac{v \gamma}{2} \lambda+(1-\eta)(1-\gamma)\left(\frac{1}{2}-z^{h l}\right)((1-\chi) \lambda-\bar{A}),
$$

which is negative if $v$ is small enough, that is,

$$
v<\frac{(1-\gamma)}{\gamma}\left(2 z^{h l}-1\right)\left(1-\chi-\frac{\bar{A}}{\lambda}\right) .
$$


In this case, Proposition 5 holds.

Q.E.D.

Proof of Proposition 9. Evaluated at $z_{i}=Q$, we have

$$
\mu(i)=\gamma>\mu(j)=\frac{(1-Q) \gamma}{\gamma+(1-\gamma)(1-Q)} .
$$

Because $\mu(i)-\mu(j)$ decreases with $z_{i}$, we obtain $z^{h l}>Q$. Then $\frac{\partial(\mu(i)-\mu(j))}{\partial Q}>0$ implies that $z^{h l}$ increases with $Q$, and hence with $v_{h} v_{l}$.

Proof of Proposition 10. We have $w^{l}>w^{h}$ because $z^{h l}>1 / 2$. Moreover,

$$
\begin{aligned}
& w^{l}=\gamma Q+(1-\gamma) z^{h l}-\frac{1}{2}+(1-\gamma) Q\left(1-z^{h l}\right)\left(\frac{1}{1+v_{h} v_{l}}-\bar{A}\right)>0, \\
& w^{h}=\gamma Q-\frac{1}{2}+(1-\gamma)\left(\frac{1+z^{h l}}{2}-\frac{1}{4} \bar{A}\right),
\end{aligned}
$$

which may be negative.

$\square \quad$ Welfare. The value $\omega^{l l}$ is unchanged. For certified lawyers, we find that

$$
\omega^{h h}=\left(1-\frac{v_{h}}{2}\right) \lambda-(1-\gamma)\left(1-v_{h}\right) \frac{1}{2} \bar{A} .
$$

Finally,

$$
\begin{aligned}
& \omega^{h l}=\left(\gamma\left(\frac{3}{2}-Q\right)+(1-\gamma)\left(1-\frac{z^{h l}}{2}\right)\right) \lambda-(1-\gamma)\left(1-z^{h l}\right) Q \bar{A} . \\
& 2 \omega^{h l}-\omega^{h h}-\omega^{l l}= \frac{\gamma}{2}\left(-2 v_{h} v_{l}+v_{l}+v_{h}\right) \lambda+(1-\gamma)\left(1-z^{h l}\right) \lambda-(1-\gamma)\left(1-v_{h}\right) \frac{1}{2} \lambda \\
&+(1-\gamma)\left(\left(1-v_{h}\right) \frac{1}{2}-\left(1-z^{h l}\right) 2 Q\right) \bar{A} .
\end{aligned}
$$

It is negative if

$$
v_{l}+v_{h}-2 v_{h} v_{l}<2\left(\frac{1-\gamma}{\gamma}\right)\left(\left(z^{h l}-\frac{1}{2}-v_{h} \frac{1}{2}\right)\left(1-\frac{\bar{A}}{\lambda}\right)+\left(1-z^{h l}\right)(2 Q-1) \frac{\bar{A}}{\lambda}\right) .
$$

\section{References}

Anderlini, L., Felli, L., And Postlewaite, A. "Courts of Law and Unforeseen Contingencies." Journal of Law, Economics, and Organization, Vol. 23 (2007), pp. 662-684.

Ashenfelter, O. And Bloom, D. "Lawyers as Agents of the Devil in a Prisoner's Dilemma Game.” Working Paper no. 4447, NBER, 1993.

Bar-IsaAC H. And TAdelis, S. "Seller Reputation." Foundations and Trends in Microeconomics, Vol. 4 (2008), pp. $273-351$.

Biglaiser, G. “Middlemen as Experts.” RAND Journal of Economics, Vol. 24 (1993), pp. 212-223.

Blanes i Vidal, J., And Leaver, C. “Pandering Judges.” Discussion Paper, University of Oxford, 2008.

Bourjade, S. AND Jullien, B. "The Roles of Reputation and Transparency on the Behavior of Biased Experts." RAND Journal of Economics, Vol. 42 (2011), pp. 575-594.

Bundy, S. And Elhauge, E. "Do Lawyers Improve the Adversary System? A General Theory of Litigation Advice and Its Regulation.” California Law Review, Vol. 79 (1991), pp. 313-420.

Che, Y. And Severinov, S. “On the Value of Legal Representation.” Mimeo, 2006.

Cooter, R. And Rubinfeld, D. "Economic Analysis of Legal Disputes and Their Resolution." Journal of Economic Literature, Vol. 29 (1989), pp. 1067-1097.

Daughety, A. And Reinganum, J. “Appealing Judgments.” RAND Journal of Economics, Vol. 31 (2000), pp. $502-526$.

Deparment of Constitution Affairs. "Constitutional Reform: The Future of Queen's Counsel." Consultation Paper no. 08/03, Department of Constitutional Affairs. 2003.

Dewatripont, M. And Tirole, J. “Advocates.” Journal of Political Economy, Vol. 107 (1999), pp. 1-39.

Ely, J. AND VÄLIMÄKI, J. “Bad Reputation.” Quarterly journal of economics, Vol. 118 (2003), pp. 785-814.

Galanter, M. "Why the "Haves" Come Out Ahead: Speculations on the Limits of Legal Change." Law \& Society Review, Vol. 9 (1974), pp. 95-160.

Garicano, L. And HubBard, T. "Specialization, Firms and Markets: The Division of Labor within and between Law Firms." Journal of Law, Economics, and Organization, Vol. 25 (2009a), pp. 339-371.

_ AND - "Earnings Inequality and Coordination Costs: Evidence from U.S. Law Firms." Working Paper no. w14741, NBER, 2009b.

Hirshleifer, J. And Osborne, E. “Truth, Effort, and the Legal Battle.” Public Choice, Vol. 108 (2001), pp. $169-195$. 
Holmström, B. “Managerial Incentive Problems: A Dynamic Perspective,” Review of Economic Studies, Vol. 66 (1999), pp. 169-182.

IossA, E. "Reputational Concerns in Arbitration: Decision Bias and Information Acquisition." Economics and Finance Discussion Papers 07-28, Brunel University, 2007.

—. AND JULLIEN, B. “The Market for Lawyers and the Quality of Legal Services.” Working Paper no. 170, CEIS, 2010. And Palumbo, G. "Information Provision and Monitoring of the Decision-Maker in the Presence of an Appeal Process." Journal of Institutional and Theoretical Economics, Vol. 163 (2007), pp. 657-682.

KAPLOW, L. AND ShaVell, S. "Legal Advice about Information to Present in Litigation: Its Effects and Social Desirability." Harvard Law Review, Vol. 102 (1989), pp. 565-615.

Leaver, C. "Bureaucratic Minimal Squawk Behaviour: Theory and Evidence from Regulatory Policy." American Economic Review, Vol. 9 (2009), pp. 572-607.

LEVY, G. “Careerist Judges.” RAND Journal of Economics, Vol. 36 (2005), pp. 275-297.

LizzERI, A. "Information Revelation and Certification Intermediaries." RAND Journal of Economics, Vol. 30 (1999), pp. 214-231.

Miceli, T. And Cosgel, M. "Reputation and Judicial Decision-Making." Journal of Economic Behavior \& Organization, Vol. 23 (1994), pp. 31-51.

Milgrom, P. AND Roberts, J. "Relying on the Information of Interested Parties." RAND Journal of Economics, Vol. 17 (1986), pp. 18-32.

Ottaviani, M. And Søerensen, P. “Reputational Cheap Talks.” RAND Journal of Economics, Vol. 37 (2006), pp. 155-175.

PAshigian, P. "The Market for Lawyers: The Determinants of the Demand for the Supply of Lawyers." Journal of Law and Economics, Vol. 20 (1977), pp. 53-85.

Prat, A. "The Wrong Kind of Transparency." American Economic Review, Vol. 95 (2005), pp. 862-877.

Rosen, S. “The Market for Lawyers.” Journal of Law and Economics, Vol. 35 (1992), pp. 215-246.

Ross, H. Settled Out of Court: The Social Process of Insurance Claims Adjustment. Chicago: Aldine, 1970.

SAUER, R. "Job Mobility and the Market for Lawyers." Journal of Political Economy, Vol. 106 (1998), pp. $147-171$.

SHAPIRO, C. "Investment, Moral Hazard, and Occupational Licensing." Review of Economic Studies, Vol. 53 (1986), pp. 843-862.

Shavell, S. "The Fundamental Divergence between the Private and the Social Motive to Use the Legal System." Journal of Legal Studies, Vol. 26 (1997), pp. 575-612.

- Foundations of Economic Analysis of Law. Cambridge, Mass.: Harvard University Press, 2004.

. "The Appeals Process and Adjudicator Incentives." Journal of Legal Studies, Vol. 35 (2006), pp. 1-29.

SHIN, H. "Adversarial and Inquisitorial Procedures in Arbitration." RAND Journal of Economics, Vol. 29 (1998), pp. 378-405.

SPIER, K. "Litigation." In A. M. Polinsky and S. Shavell, eds., The Handbook of Law and Economics, Vol.1. Oxford: North Holland 2007.

SPURR, S. "How the Market Solves an Assignment Problem: The Matching of Lawyers with Legal Claims." Journal of Labor Economics, Vol. 5 (1987), pp. 502-532. 